\title{
Function approximation on arbitrary domains using Fourier extension frames
}

\author{
Roel Matthysen \\ Daan Huybrechs
}

Report TW 679, June 9, 2017

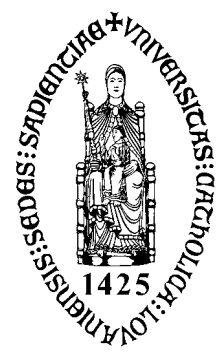

\section{KU Leuven}

Department of Computer Science

Celestijnenlaan 200A - B-3001 Heverlee (Belgium) 


\title{
Function approximation on arbitrary domains using Fourier extension frames
}

\author{
Roel Matthysen \\ Daan Huybrechs
}

Report TW679, June 9, 2017

\author{
Department of Computer Science, KU Leuven
}

\begin{abstract}
Fourier extension is an approximation scheme in which a function on an arbitary bounded domain is approximated using a classical Fourier series on a bounding box. On the smaller domain the Fourier series exhibits redundancy, and it has the mathematical structure of a frame rather than a basis. It is not trivial to construct approximations in this frame using function evaluations in points that belong to the domain only, but one way to do so is through a discrete least squares approximation. The corresponding system is extremely ill-conditioned, due to the redundancy in the frame, yet its solution via a regularized SVD is known to be accurate to very high (and nearly spectral) precision. Still, this computation requires $\mathcal{O}\left(N^{3}\right)$ operations. In this paper we describe an algorithm to compute such Fourier extension frame approximations in only $\mathcal{O}\left(N^{2} \log ^{2} N\right)$ operations for general 2D domains. The cost improves to $\mathcal{O}\left(N \log ^{2} N\right)$ operations for simpler tensor-product domains. The algorithm exploits a phenomenon called the plunge region in the analysis of time-frequency localization operators, which manifests itself here as a sudden drop in the singular values of the least squares matrix. It is known that the size of the plunge region scales like $\mathcal{O}(\log N)$ in one dimensional problems. In this paper we show that for most $2 \mathrm{D}$ domains in the fully discrete case the plunge region scales like $\mathcal{O}(N \log N)$, proving a discrete equivalent of a result that was conjectured by Widom for a related continuous problem. The complexity estimate depends on the Minkowski or box-counting dimension of the domain boundary, and as such it is larger than $\mathcal{O}(N \log N)$ for domains with fractal shape.

Keywords : Fourier Series, Fourier Extensions, Fourier Continuation, Prolate Spheroidal Wave Theory.
\end{abstract}




\title{
Function approximation on arbitrary domains using Fourier extension frames
}

\author{
Roel Matthysen, Daan Huybrechs
}

June 8, 2017

\begin{abstract}
Fourier extension is an approximation scheme in which a function on an arbitary bounded domain is approximated using a classical Fourier series on a bounding box. On the smaller domain the Fourier series exhibits redundancy, and it has the mathematical structure of a frame rather than a basis. It is not trivial to construct approximations in this frame using function evaluations in points that belong to the domain only, but one way to do so is through a discrete least squares approximation. The corresponding system is extremely ill-conditioned, due to the redundancy in the frame, yet its solution via a regularized SVD is known to be accurate to very high (and nearly spectral) precision. Still, this computation requires $\mathcal{O}\left(N^{3}\right)$ operations. In this paper we describe an algorithm to compute such Fourier extension frame approximations in only $\mathcal{O}\left(N^{2} \log ^{2} N\right)$ operations for general 2D domains. The cost improves to $\mathcal{O}\left(N \log ^{2} N\right)$ operations for simpler tensor-product domains. The algorithm exploits a phenomenon called the plunge region in the analysis of time-frequency localization operators, which manifests itself here as a sudden drop in the singular values of the least squares matrix. It is known that the size of the plunge region scales like $\mathcal{O}(\log N)$ in one dimensional problems. In this paper we show that for most $2 \mathrm{D}$ domains in the fully discrete case the plunge region scales like $\mathcal{O}(N \log N)$, proving a discrete equivalent of a result that was conjectured by Widom for a related continuous problem. The complexity estimate depends on the Minkowski or box-counting dimension of the domain boundary, and as such it is larger than $\mathcal{O}(N \log N)$ for domains with fractal shape.
\end{abstract}

\section{Introduction}

The nature of the discrete representation of a continuous function is an important choice in many applications. A representation needs to be efficiently constructed as well as easy to use. The number of degrees of freedom in the representation influences both of these considerations. We focus on methods that offer a high rate of convergence for increasing degrees of freedom, specifically converging at least superalgebraically.

In two dimensions and higher, the domain of the function is an important complication in the approximation process. When the domain has some structure, such as a rectangle, it is possible to modify existing one-dimensional spectral or high order methods to fit the domain. When the domain shape is arbitrary, in many cases one resorts to a polygonal mesh with basis functions that have low orders of smoothness. Radial Basis Functions are well suited for unstructured data and may offer spectral accuracy, but they typically require dense matrix computations and are dependent on well-chosen shape parameters [14].

The representation used in this paper is based on orthogonal basis functions on a bounding box that encloses the domain. The objective is to obtain an expansion in this basis that resembles a given function as closely as possible on the domain. An example of a possible resulting approximation is shown in fig. 1, which contains all the main elements of this procedure. In fig. 1a the function $f(x, y)=\cos \left(20 x^{2}-15 y^{2}\right)$ is shown, where the domain takes on the shape of Belgium. This function is approximated using Fourier basis functions on a bounding rectangle. Therefore the expansion in 
fig. $1 \mathrm{~b}$ is periodic. Such an approximation is straightforward to compute when the function to be approximated can be evaluated throughout the bounding box. Yet, the approximation problem itself becomes challenging when one is restricted to function samples in the irregular domain only, and that is the setting we pursue.

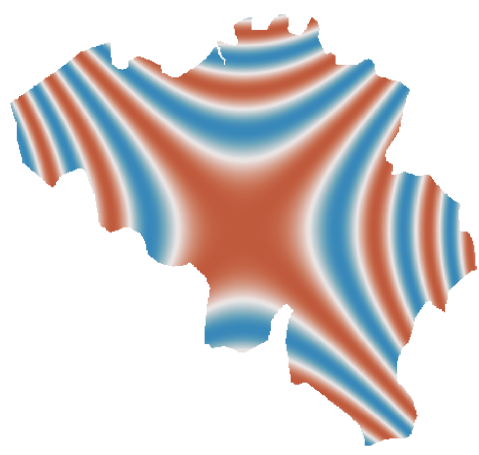

(a) Data points

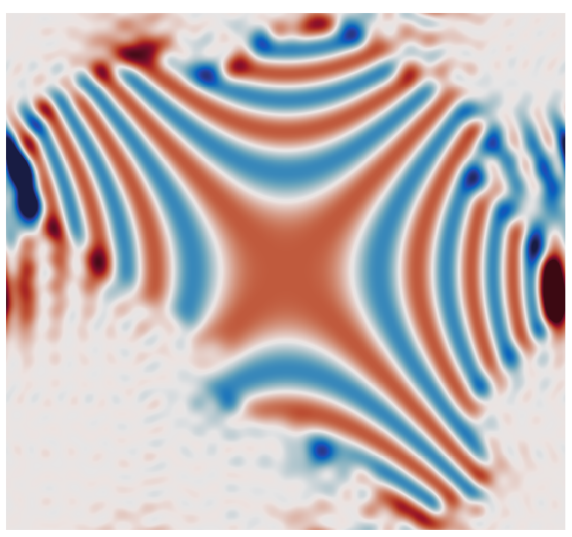

(b) Fourier Series on bounding box

Figure 1: Approximation of $f(x, y)=\cos \left(20 x^{2}-15 y^{2}\right)$ on a Belgium-shaped domain, using a Fourier series on a bounding box.

This method for one-dimensional Fourier bases is known as the Fourier extension (FE) or Fourier continuation (FC) technique $[6,7,8,9,15]$. In this technique the approximation is computed by performing a least squares approximation on an equispaced grid of collocation points. This allows the use of the FFT, which facilitates fast algorithms. The main problem is the ill-conditioning of the collocation matrix.

The FE method is closely related to embedded or fictitious domain methods for solving certain partial differential equations using Fourier basis functions, the main difference being the approximation in the extension region. In embedded domain methods the function is explicitly extended outside the domain of interest, e. g. through convolution with gaussian kernels [11] or using polynomial corrections [26]. In the Fourier extension technique, the approximation in the extension region is determined implicitly through solving a least squares problem.

Convergence properties of $\mathrm{FE}$ for function approximation were first described in [6] and [8]. It was shown that a Fourier basis approximation can converge exponentially to $f$ inside the domain of interest. The approximation problem can be solved using discrete least squares and a truncated singular value decomposition. Bruno and Pohlmann used the FE technique in higher dimensions to obtain smooth and periodic extensions around boundaries of complicated surfaces. This was the basis for the very efficient FC-Gram method for approximations and solving differential equations, using 1D Fourier extensions combined with an ADI approach [10, 23, 4]. Analysis of Fourier extensions continued in the context of frames, with precise convergence rates and error bounds now available $[15,1,2,3]$. A major conclusion in the latter references is that, in spite of potentially extreme ill-conditioning, the regularized SVD solution of the discrete problem is numerically stable once sufficiently many degrees of freedom are used. Furthermore, a discrete least squares is more accurate than projection methods using the Gram matrix. This is true not only for Fourier extension, but more generally for numerical approximations in frames [3].

Although the FE technique is very flexible when it comes to domain choice and yields fast converging approximations, computing the expansion is not efficient. The singular value decomposition is cubic in the number of degrees of freedom, making it very slow for even mildly oscillatory functions. In [22] and [24], two distinct $O\left(N \log ^{2} N\right)$ algorithms for the 1D approximation scheme were introduced, where $N$ is the number of degrees of freedom. 
The $O\left(N \log ^{2} N\right)$ complexity of the latter reference [24] originates in the connection of this problem to Prolate Spheroidal Wave theory [32, 18, 27]. It allows for an interpretation of the singular values and vectors of the problem, in terms of functions that are maximally concentrated simultaneously in time and frequency. This is a topic in signal processing mostly, but in our context it shows that the singular values can be separated into three clusters: one cluster of values is exponentially close to 1 , a second one is exponentially close to 0 , and a third transitional set in between contains values exponentially dropping from 1 to machine precision. This very particular distribution of singular values is illustrated in fig. 3 further on and is seen for domains of any shape. The latter transitional set is called the plunge region. Crucially, the size of this plunge region has smaller complexity than the other clusters: it is known for one-dimensional problems that it grows only as $O(\log N)$ [36]. The fast algorithm of [24] is based on a projection of the problem onto a smaller one, with dimensions governed by the size of the plunge region, which is solved with a direct method. It is followed by a post-processing step that amounts to a single FFT. Details are recalled in section 2 of this paper. In spite of its mathematical intricacies, the algorithm itself is short and simple, and a full implementation for the 1D case was included in the appendix of [24].

It is clear that the cost of the algorithm is directly influenced by the size of the plunge region. Unfortunately, the plunge region for $2 \mathrm{D}$ domains is relatively larger than it is in 1D. For tensorproduct domains, the tensor product structure can be readily exploited to generalize the algorithm of [24] with near-optimal complexity. For other domains, somewhat surprisingly, the plunge-projection method still applies virtually without modification. However, the size of the plunge region has not received as much study as in the one-dimensional case. In this paper we establish the size of the plunge region, and, hence, the computational cost of the algorithm. Loosely speaking, the plunge region scales with the size of the boundary of the domain, rather than with the domain itself. As such, it is a lower-dimensional phenomenon, which we confirm in this paper for the $2 \mathrm{D}$ case $^{1}$.

\subsection{Overview and main results of the paper}

In section 2 of this paper we describe the application of the $1 \mathrm{D}$ algorithm to the multidimensional case, and we state the conditions that are necessary for a speedup compared to the full direct solver with cubic cost. This entails a proper choice of sample points in time and frequency.

In section 3 we show a bound on the singular value distribution of the collocation matrix. As indicated above, this corresponds to an estimate of the size of the plunge region for the discrete Fourier transform, for two-dimensional domains in the time domain and a rectangular region in the frequency domain. This bound is a direct generalization of the discrete time-frequency localization results of Wilson in [36], and are a discrete analog of recent results by Sobolev [33, 34] on a related conjecture by Widom [35]. Most of our results, but not all, are formulated for a general dimension $D$.

Section 4 contains numerical results for approximations on a variety of domains in a Fourier basis, demonstrating the flexibility, accuracy and speed of our approach.

The contribution of this paper is twofold: we show the accuracy and reliability of Fourier frame approximations in higher dimensions, while providing a deeper understanding of the spectral properties of the problem through explicit bounds. This leads to an algorithm that is asymptotically faster than a direct solver on a rank-deficient rectangular least squares problem.

\section{Problem formulation}

\subsection{Approximation in a Fourier frame}

Informally, the problem formulation is as follows: given a function $f$ and a domain $\Omega \subset \mathbb{R}^{D}$, find a Fourier series $F$ that minimizes a suitable norm $\|F-f\|_{X}$ on $\Omega$, using only information about $f$ on $\Omega$.

\footnotetext{
${ }^{1}$ Julia code for this algorithm is incorporated in the FrameFun package, at http://github.com/daanhb/FrameFun.jl
} 
Let us elaborate and be precise. Without loss of generality, assume that $\Omega \subset R=[0,1]^{D}$, so that we can use a tensor-product of the standard Fourier series on $[0,1]$. In order to avoid any periodicity requirements on $f$, we assume further that $\Omega$ lies fully in the interior of the box $R$. In the following, we will consistently use the symbols $\Omega$ for the time domain, and $\Lambda$ in the frequency domain. For an index set $P_{\Lambda}$ with $N_{\Lambda}$ frequencies, we denote the basis functions and the function space they span by

$$
\begin{aligned}
\phi_{\mathbf{1}}(\boldsymbol{x}) & =e^{\mathrm{i}(\boldsymbol{x} \cdot \mathbf{l}) 2 \pi}, \\
\mathcal{G}_{N_{\Lambda}} & =\operatorname{span}\left\{\phi_{\mathbf{1}}\right\}_{\mathbf{l} \in P_{\Lambda}} .
\end{aligned}
$$

Here, $\boldsymbol{x}=\left(\boldsymbol{x}_{1}, \ldots, \boldsymbol{x}_{D}\right)$ is a $D$-dimensional point and $\mathbf{l}=\left(\mathbf{l}_{1}, \ldots, \mathbf{l}_{D}\right)$ is a $D$-dimensional integer index. For simplicity, we assume an equal number of degrees of freedom $n_{\Lambda}$ per dimension, hence $N_{\Lambda}=n_{\Lambda}^{D}$ and $\left\lfloor n_{\Lambda} / 2\right\rfloor<\mathbf{l}_{i}<\left\lceil n_{\Lambda} / 2\right\rceil$. This restriction could be lifted at the cost of minor complications further on, and we do not make this assumption in our implementation.

The approximation problem in this space is stated as

$$
F_{N_{\Lambda}}(f)=\min _{g \in \mathcal{G}_{N_{\Lambda}}}\|f-g\|_{X}
$$

Note here that the function set $\mathcal{G}$ restricted to $\Omega$ is not a basis for $\mathcal{L}^{2}(\Omega)$, but it is a frame in the sense of Duffin and Schaeffer [12]. For a recent overview of numerical frame approximations, see [3].

Any $g \in \mathcal{G}$ is uniquely described by a set of coefficients $\mathbf{a} \in \mathbb{C}^{n_{\Lambda} \times \cdots \times n_{\Lambda}}$. In the remainder we will often assume an implicit linearization $\mathbf{a} \in \mathbb{C}^{N_{\Lambda}}$. These coefficients will be the result of the approximation algorithm, so we look for

$$
\mathbf{a}=\underset{\mathbf{c} \in \mathbb{C}^{N_{\Lambda}}}{\arg \min }\left\|f-\sum_{\mathbf{l} \in P_{\Lambda}} \mathbf{c}_{\mathbf{l}} \phi_{\mathbf{l}}\right\|_{X}
$$

It remains to define the norm $\|\cdot\|_{X}$. The choice of the $\mathcal{L}^{2}$ norm over $\Omega$ is fairly natural, and this choice leads to the so-called Continuous Frame approximation. The minimizer of eq. (4) is found by constructing the Gram matrix

$$
A_{\mathbf{k}, \mathbf{l}}^{G}=\left\langle\phi_{\mathbf{k}}, \phi_{\mathbf{l}}\right\rangle_{\Omega}
$$

and solving the system

$$
A^{G} \mathbf{a}=b, \quad b_{\mathbf{l}}=\left\langle f, \phi_{\mathbf{l}}\right\rangle_{\Omega} .
$$

However, the computational cost associated with evaluating the integrals in the right hand side of (6) is considerable, since the integrals are over $\Omega$ and not over the full box $R$. This precludes the use of the FFT and one would have to resort to some type of quadrature on $\Omega$.

Instead, in this paper we will focus on the Discrete Frame approximation. The corresponding norm is a discrete summation over a set of collocation points. For Fourier approximations, we choose a set of equispaced points $P_{R}$ on $R$ (recall that $R=[0,1]^{D}$ ) with $n_{R}$ points per dimension, and restrict those to $\Omega$. In summary

$$
\left.P_{R}=\left\{\left(\frac{k_{1}}{n_{R}}, \ldots, \frac{k_{D}}{n_{R}}\right)\right) \mid \forall i: 0 \leq k_{i}<n_{R}\right\}, \quad P_{\Omega}=P_{R} \cap \Omega .
$$

There are efficient transformations using the FFT between the set $P_{R}$ of $N_{R}=n_{R}^{D}$ points in the time domain and the set $P_{\tilde{R}}$ in the frequency domain. If we choose $n_{R} \geq n_{\Lambda}$, then $P_{\tilde{R}}$ encompasses $P_{\Lambda}$. The sampling sets thus defined are shown in fig. 2. Though other choices can be made, this choice is such that we can efficiently evaluate a Fourier series using the index set $P_{\Lambda}$ in all the points of $P_{\Omega}$ : we extend the coefficients with zeros from $P_{\Lambda}$ to $P_{\tilde{R}}$, followed by an FFT transform from $P_{\tilde{R}}$ to $P_{R}$, followed by a restriction of the values to those points in $P_{\Omega}$.

Throughout this paper we assume a fixed oversampling rate, meaning $N_{\Lambda} / N_{\tilde{R}}$ is constant. We refer to [?] for a study on the interplay of oversampling rate and choice of bounding box in one dimension. 


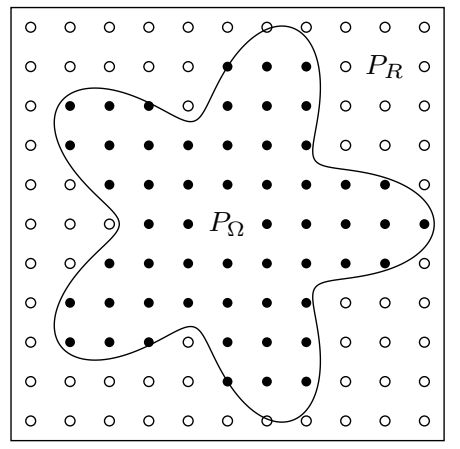

(a) time domain

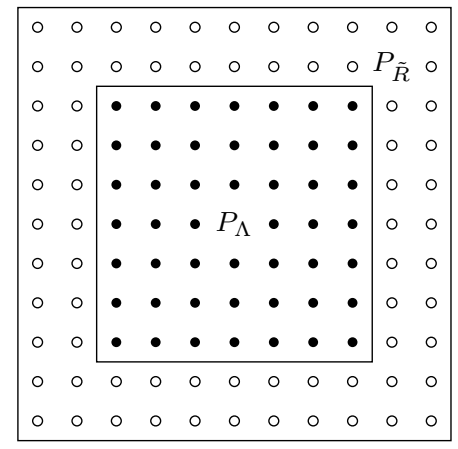

(b) frequency domain

Figure 2: The spatial domain $\Omega$ encompassing the sample set $P_{\Omega}$, and the frequency domain $\Lambda$ encompassing the discrete frequencies $P_{\Lambda}$. There is a fast FFT transform between the encompassing sets $P_{R}$ and $P_{\tilde{R}}$.

The minimization (4) can be reformulated as a discrete least squares problem

$$
F_{N_{\Lambda}}(f)=\underset{g \in \mathcal{G}_{N_{\Lambda}}}{\arg \min } \sum_{\boldsymbol{x} \in P_{\Omega}}(f(\boldsymbol{x})-g(\boldsymbol{x}))^{2} .
$$

Assuming a linear indexing $\boldsymbol{x}_{k}$ of $P_{\Omega}$ from 1 to $N_{\Omega}$ and $\phi_{j}$ of $P_{\Lambda}$ from 1 to $N_{\Lambda}$, it can be written as a least squares matrix problem

$$
A \mathbf{a}=b, \quad A \in \mathbb{C}^{N_{\Omega} \times N_{\Lambda}}, \quad b \in \mathbb{C}^{N_{\Omega}}
$$

where

$$
A_{k j}=\frac{1}{\sqrt{N_{R}}} \phi_{j}\left(\boldsymbol{x}_{k}\right), \quad b_{k}=f\left(\boldsymbol{x}_{k}\right) .
$$

The scaling of the basisfunctions is such that $A$ is precisely a subblock of a multidimensional unitary DFT matrix, which is of importance to algorithm 1. This subblock property is a consequence of our choice of discrete grids, and it results in a fast matrix-vector product using the procedure described above: extension in frequency domain, discrete Fourier transform, and restriction in the time domain. Indeed, note that in this discrete setting the action of the matrix $A$ corresponds to evaluating a length $N_{\Lambda}$ Fourier series in the points of $P_{\Omega}$.

Note that there is also a fast matrix-vector product for $A^{\prime}$ and it corresponds to the opposite sequence of operations: extension from $P_{\Omega}$ to $P_{R}$ by zeros, fast transform to $P_{\tilde{R}}$, followed by restriction in the frequency domain from $P_{\tilde{R}}$ to $P_{\Lambda}$. Yet, it is clear that the solution to $A x=b$ is not simply given by $x=A^{\prime} b$ : the latter is accurate only when the extension of the function $f$ by zero is well approximated by a Fourier series, which in general it is not. Extension by zero in the time domain introduces a discontinuity. On the other hand, extending a Fourier series with additional zero coefficients does not affect the function it describes.

Remark 1. Though the examples shown use Fourier bases exclusively, a Chebyshev collocation matrix in Chebyshev points is entirely analogous. It consists of a scaled subblock of a multidimensional DCT matrix. Therefore, most of the arguments, although not made explicit in this paper, apply to this case as well. This is of course due to the close connection between Chebyshev polynomials and trigonometric polynomials.

\subsection{An approximation algorithm by projection onto the plunge region}

Equation (9) corresponds to a dense, rectangular linear system that is rank-deficient: the condition number of $A$ is exponentially large. Yet, high accuracy can be achieved with direct solvers such as a 
pivoted QR decomposition ( MATLAB 's backslash). Iterative algorithms, on the other hand, are much less suitable, in spite of a fast matrix-vector product being available, due to the ill-conditioning.

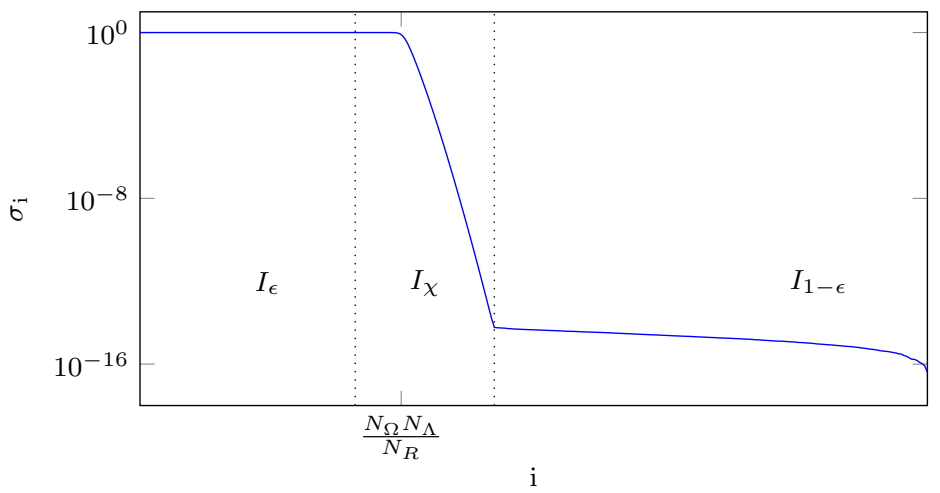

Figure 3: The subdivision of the spectrum of $A$ into three distinct intervals, with cutoff parameter $\tau=1 e-14$. The singular values cluster near 1 and 0 , and there is a plunge region in between. Due to rounding errors, the eigenvalues in region $I_{\epsilon}$ don't decay past machine precision.

Still, matrix $A$ has a lot of structure that we set out to exploit. The singular values have a very distinct profile, shown in fig. 3 . There is a region $I_{1-\epsilon}$ of singular values that are close to 1 up to a small value $\epsilon$, but never exceeding it. Its size, as proven in section 3 , is approximately $\frac{N_{\Omega} N_{\Lambda}}{N_{R}}$. There is a similar region $I_{\epsilon}$ that contains singular values smaller than $\epsilon$. Inbetween there is a plunge region $I_{\chi}$, which contains singular values between $\epsilon$ and $1-\epsilon$.

Denote by $\eta\left(\epsilon, N_{R}\right)$ the size of the plunge region $I_{\chi}$,

$$
\eta\left(\epsilon, N_{R}\right)=\#\left\{\sigma_{k}: \epsilon<\sigma_{k}<1-\epsilon\right\} .
$$

For the 1D discrete Fourier extension problem this quantity is known to grow slowly with $N_{R}[36]$

$$
\eta\left(\epsilon, N_{R}\right)=O\left(\log \left(N_{R}\right)\right) .
$$

Intuitively, one could state that just $\eta\left(\epsilon, N_{R}\right)$ of the singular values capture almost all the ill-conditioning present in $A$. This observation led to an algorithm for eq. (9) that is $O\left(N_{R} \eta\left(\epsilon, N_{R}\right)^{2}\right)=O\left(N_{R} \log ^{2}\left(N_{R}\right)\right)$ [24], which we now recall in some detail. Note that due to our definitions, $N_{\Lambda}$ asymptotically grows proportionally to $N_{R}$, hence the complexities in terms of $N_{\Lambda}$ and of $N_{R}$ are equivalent.

The algorithm of [24] solves the system eq. (9) under the assumption that a solution with sufficiently small norm $\|x\|$ and small residual $A x-b$ exists. We refer to [2] for conditions on the existence of these solutions in the 1D Fourier extension case, and [3] for a detailed treatment in the general context of frames.

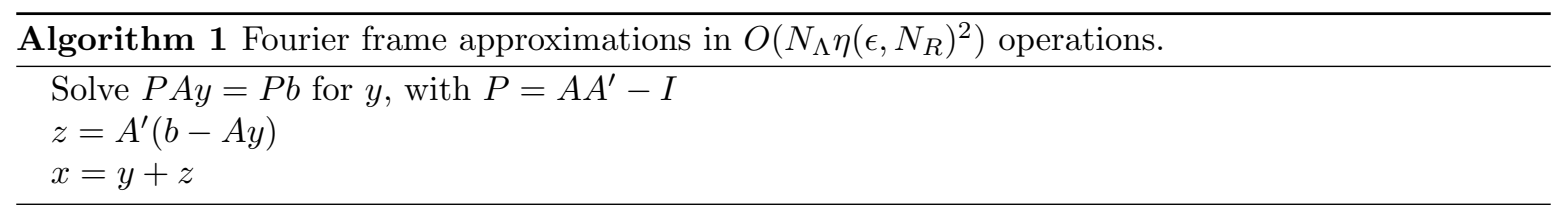

The algorithm is simple to state, and it is included schematically in algorithm 1. Its steps do require some more explanation:

1. The $\eta\left(\epsilon, N_{R}\right)$ singular values in the middle region can be isolated by multiplying both $A$ and $b$ with a matrix $P=A A^{\prime}-I$. This multiplication maps the singular values $\sigma$ of $A$ to $\sigma^{3}-\sigma$, since 
if $A=U \Sigma V^{\prime}$ then $\left(A A^{\prime}-I\right) A=U\left(\Sigma^{3}-\Sigma\right) V^{\prime}$. This effectively removes all singular values close to either one or zero. As a result, the linear system

$$
P A y=P b
$$

has numerical rank $\chi\left(\epsilon_{\text {mach }}, N_{R}\right)$, and a solution $y$ can be obtained in $O\left(N_{R} \log ^{2}\left(N_{R}\right)\right)$ operations, e. g. through randomized algorithms [21].

2. The partial solution vector $y$ has a residual $r=b-A y$. If we find a vector $z$ such that $A z=r$, then $x=y+z$ solves the overall problem. Indeed, in that case $A x=A y+A z=b-r+r=b$.

3. Crucially, the problem for $z$ is simple to solve, $z=A^{\prime} r=A^{\prime}(b-A y)$. If an accurate solution exists, then $r$ must lie in the column space of $A$. Since $r$ is by construction orthogonal to the column space associated with $I_{\chi}$, it must in fact lie in the space associated with $I_{\epsilon}$. Yet, since the corresponding singular values are close to 1 , in this subspace the inverse of $A$ is well approximated by its adjoint $A^{\prime}$.

The correctness of the algorithm is shown formally in [24]. For the purpose of the present article, here we make the following observations:

- A fast matrix-vector product with $A$ and $A^{\prime}$ is available in any dimension, regardless of the shape of the domain $\Omega$.

- Algorithm 1 is purely algebraic and applies to any system $A x=b$, as long as $A$ has a singular value profile similar to the one shown in fig. 3, exhibiting a plunge region from 1 to 0.

- The computational cost of the algorithm depends quadratically on the size of the plunge region: it is $O\left(N_{\Lambda} \eta\left(\epsilon, N_{R}\right)^{2}\right)$ operations.

We show numerical results using Algorithm 1 for a variety of domains in section 4. Compared to its univariate implementation described in [24], the single conceptual complication lies in the suitable identification of the sampling sets as illustrated in fig. 2. Mathematically, however, determining the size of the plunge region - and with it the computational complexity of our algorithm - is significantly more involved. The next section contains a brief historical context of the literature on the asymptotic behaviour of $\eta\left(\epsilon, N_{R}\right)$, as there has been quite some interest in the equivalent continuous problem.

\section{Spectrum of the collocation matrix}

\subsection{One dimensional bandlimited extrapolation}

We begin this section by highlighting the close interconnection between the Fourier frame approximation problem and that of bandlimited extrapolation in classical signal processing literature. Much attention has been given to the problem of extending or extrapolating a function that is known to be bandlimited, from limited data. The ingredients of the two problems, a truncated basis and limited data, are the continuous equivalents of fig. 2 , and as such it is not surprising that the methods have a lot in common.

The theory on bandlimited extrapolation was pioneered in a series of papers by Slepian and collaborators in the 1960s and 1970s [32] [18] [27] [28]. Specifically, they studied the integral equation

$$
\lambda_{i} \psi_{i}(s)=\int_{\Omega} \psi_{i}(t) \frac{\sin W(t-s)}{\pi(t-s)} d t
$$

whose solutions are called prolate spheroidal wave functions. This equation corresponds to the question To what extent $\lambda_{i}$ can a function be concentrated both in the time- and frequency domain?. Indeed, in this equation the function has finite bandwidth $W$ and the equation expresses that a fraction $\lambda_{i}$ of the 
energy of $\psi_{i}$ is contained in the subdomain $\Omega \subset \mathbb{R}$. In what is known as the uncertainty principle, or the Gabor limit, they showed that the eigenvalues approach but never exactly equal 1, and so when properly ordered,

$$
1>\lambda_{1}>\lambda_{2}>\cdots>0 \text {. }
$$

Moreover, the eigenvalues cluster near 1 and zero as $W \rightarrow 0$, or equivalently, as the frequency limit increases. That is, for any small $\epsilon$, the number of eigenvalues between $1-\epsilon$ and $\epsilon$ grows like $O\left(\log W^{-1}\right)$.

Following up on this result, Slepian defined and proved similar results for a discrete version of the prolate spheroidal wave functions [31]. In this case the frequency domain is sampled at regular intervals, and the sinc kernel is replaced by a Dirichlet kernel. Equation (14) becomes

$$
\lambda_{i} \psi_{i}(s)=\int_{\Omega} \psi_{i}(t) \frac{\sin W(t-s)}{\sin (\pi(t-s))} d t .
$$

The eigenfunctions and corresponding Fourier series coefficients are optimally concentrated in a continuous time and discrete frequency domain, or vice versa. The clustering property was proven as well.

When both time and frequency domain are discrete, the resulting sequences viewed on their domain of restriction become finite vectors [16]. Equation (15) becomes a difference equation [37]:

$$
\sum_{n=0}^{M} \frac{\sin ((2 K+1)(m-n) \pi / N)}{N \sin ((m-n) \pi / N)} \psi_{i}[n]=\lambda_{i} \psi_{i}[n] .
$$

These periodic discrete prolate spheroidal sequences $\psi_{i}[n]$ where later proven by Wilson to have the same asymptotic scaling of $\eta(\epsilon, N)$ as their continuous counterparts [36]. This result is sufficient to establish the asymptotic complexity of algorithm 1 in one dimension.

Another generalization of the results by Slepian and his collaborators came by viewing eq. (14) as a special case of a more general Wiener-Hopf operator $\left(T_{\alpha} \psi\right)(\boldsymbol{x})$

$$
\left(T_{\alpha} \psi\right)(\boldsymbol{x})=\left(\frac{\alpha}{2 \pi}\right)^{D} \chi_{\Lambda}(\boldsymbol{x}) \int_{\Omega} \int_{\Lambda} e^{i \alpha \boldsymbol{\xi} \cdot(\boldsymbol{x}-\boldsymbol{y})} \psi(\boldsymbol{y}) d \boldsymbol{y} d \boldsymbol{\xi}, \alpha>0 .
$$

This operator in $L^{2}\left(\mathbb{R}^{d}\right)$ can be reduced to eq. (14) by taking $\Lambda$ and $\Omega$ intervals in $\mathbb{R}$ and setting $\alpha \sim W^{-1}$. Due to the characteristic functions of $\Omega$ and $\Lambda$, these operators have a discontinuous symbol. Considerable effort has gone into describing the spectral properties of these operators. Starting with Slepian and Pollack [27], the eigenvalue distribution has been deduced from the trace of functions of the operators. They showed that for (14)

$$
\lim _{\alpha \rightarrow \infty} \operatorname{Tr}\left(T_{\alpha}\right)=\lim _{\alpha \rightarrow \infty} \sum_{i}^{\infty} \lambda_{\alpha, i}=C_{0} \alpha+O(1)
$$

and

$$
\lim _{\alpha \rightarrow \infty} \operatorname{Tr}\left(T_{\alpha}^{2}\right)=\lim _{\alpha \rightarrow \infty} \sum_{i}^{\infty} \lambda_{\alpha, i}^{2}=C_{0} \alpha+C_{1} \log \alpha+O(1) .
$$

Combining these traces with (15) a combinatorial argument (which we will return to in Theorem 8) shows that $\eta(\epsilon, \alpha)$ only grows as $\log \alpha$. This technique, utilizing the trace of $T_{\alpha}-T_{\alpha}^{2}$, was later used by Landau and Widom to prove equivalent results when $\Omega$ consists of a finite number of distinct intervals [19].

\subsection{Multi-dimensional extensions}

While the one dimensional Prolate Spheroidal Wave functions received considerable interest in signal processing and mathematics [25], the generalization to multiple dimensions is not straightforward. 
Most generalizations are restricted to a setting where $\Omega=\Lambda$, or require at least some structure in both time and frequency domains. In contrast, the most general multidimensional equivalent of (18) would be for arbitrary 'frequency' and 'time' domains.

Multidimensional equivalents of PSWFs were first considered by Slepian and Pollack [28]. They proved a double orthogonality property similar to the one dimensional case, and an eigenvalue distribution as in eq. (15). Afterwards, they focused only on the most symmetric case, where both $\Omega$ and $\Lambda$ are circular. In this case, the symmetry of the problem leads to PSWF generalizations as a combination of Bessel functions and one-dimensional PSWFs. Later results were described for rectangular time and frequency domains [5], or circular frequency regions [29]. For an overview, see [30].

Results on spectral properties for arbitrarily shaped regions appeared in 1982 [35], when H. Widom stated a conjecture on the traces of functions of Wiener-Hopf operators with discontinuous symbols in higher dimensions. He conjectured that an operator $T_{\alpha}$ as in eq. (18) for higher-dimensional $\Omega$ and $\Lambda$ would obey the trace relation

$$
\lim _{\alpha \rightarrow \infty} \operatorname{Tr}\left(T_{\alpha}-T_{\alpha}^{2}\right)=\alpha^{d-1} \log \alpha \mathcal{W}_{1}(\delta \Lambda, \delta \Omega)+o\left(\alpha^{d-1} \log \alpha\right) .
$$

Combined with

$$
\operatorname{Tr}\left(T_{\alpha}\right)=\left(\frac{\alpha}{2 \pi}\right)^{d} \int_{\Omega} \int_{\Lambda} d \boldsymbol{\xi} d \boldsymbol{x}
$$

this yields a plunge region that grows at least one order slower in $\alpha$ than the region of ones (up to a log-factor). Moreover, the constant

$$
\mathcal{W}_{1}(\Lambda, \Omega)=\frac{1}{2(2 \pi)^{d+1}} \int_{\delta \Lambda} \int_{\delta \Omega}\left|\boldsymbol{n}_{\delta \Lambda}(\boldsymbol{x}) \cdot \boldsymbol{n}_{\delta \Omega}\right| d \boldsymbol{\xi} d \boldsymbol{x}
$$

is dependent only on the geometry of the domains $\Omega$ and $\Lambda$. This conjecture was proven in 2010 by Sobolev [33] for arbitrary smooth domains, and the proof was later extended to piecewise continuous domains [34].

\subsection{Generalizing discrete Prolate Spheroidal wave sequences}

In light of the work by Slepian et. al., and as already alluded to in section 2 of this paper, the matrix $A$ in our fully discrete setting can be seen as the composition of three operations in time and frequency: extending $\Lambda$ to $\tilde{R}$ by zeros in the frequency domain, applying a discrete Fourier transform and restricting the result to $\Omega$ in the time domain. We shall develop this notion more formally.

We introduce several operators, which operate on sequences of length $N_{R}$ on an $n_{R} \times n_{R} \times \ldots$ grid. For indexing purposes we convert eq. (7) to the integer sets

$$
I_{R}=n_{R} P_{\Omega} \quad \text { and } \quad I_{\Omega}=n_{R} P_{\Omega} .
$$

We denote by $T_{\Omega}$ the discrete space-limiting operator that sets all values outside $\Omega$ to zero,

$$
\left(T_{\Omega}\right)_{\mathbf{k}, \mathbf{l}}=\left\{\begin{array}{cc}
1, & \mathbf{k}=\mathbf{l} \in I_{\Omega} \\
0 & \text { otherwise }
\end{array}\right.
$$

Similarly, the discrete operator $B_{\Lambda}$ is an $N_{\tilde{R}} \times N_{\tilde{R}}$ bandlimiting operator that eliminates all frequency content outside $\Lambda$. With $F$ the $D$-dimensional Fourier transform, $B_{\Lambda}=F T_{\Lambda} F^{*}$.

With these definitions, the matrix $A A^{\prime}$ is the nonzero subblock of the operator $T_{\Omega} B_{\Lambda} T_{\Omega}$. Similar to the univariate case in [24], the entries of $T_{\Omega} B_{\Lambda} T_{\Omega}$ are given in terms of a convolution kernel

$$
\left(T_{\Omega} B_{\Lambda} T_{\Omega}\right)_{\mathbf{k}, \mathbf{l}}=B(\mathbf{k}-\mathbf{l}), \quad \forall \mathbf{k}, \mathbf{l} \in I_{\Omega}
$$


where in the multivariate case $B$ is a product of univariate Dirichlet kernels,

$$
\begin{aligned}
B(\mathbf{k}) & =\prod_{d=1}^{D} b\left(k_{d}\right) \\
b(k) & =\frac{\sin \left(\pi n_{\Lambda} k / n_{R}\right)}{n_{R} \sin \left(\pi k / n_{R}\right)} .
\end{aligned}
$$

Here, $\mathbf{k}=\left(k_{1}, k_{2}, \ldots\right)$ can be a multidimensional point. Recall that $D$ is the number of dimensions.

Denote the eigenvectors of the related Hermitian matrix $B_{\Lambda} T_{\Omega} B_{\Lambda}$ by $\phi_{i}$ and by $\hat{\phi}_{i}=T_{\Omega} \phi_{i}$ the eigenvectors of $T_{\Omega} B_{\Lambda} T_{\Omega}$. The corresponding eigenvalues of both matrices are the same and denoted by $\lambda_{i}$. Similar to $[16,37,36]$, the following properties can be shown:

1. The eigenvalues are bounded above by 1 and below by 0 .

2. The rank of $T_{\Omega} B_{\Lambda} T_{\Omega}$ and of $B_{\Lambda} T_{\Omega} B_{\Lambda}$ is $\min \left(N_{\Lambda}, N_{\Omega}\right)$.

3. If $N_{\Lambda}<N_{\Omega}$, the $\phi_{i}$ are complete in the space of sequences bandlimited in $\Lambda$.

4. Define the discrete inner products $\left\langle\phi_{i}, \phi_{j}\right\rangle_{R}=\phi_{i} \cdot \phi_{j}$ and $\left\langle\phi_{i}, \phi_{j}\right\rangle_{\Omega}=\left(T_{\Omega} \phi_{i}\right) \cdot\left(T_{\Omega} \phi_{j}\right)$. The $\phi_{i}$ are doubly orthogonal with respect to these inner products,

$$
\left\langle\phi_{i}, \phi_{j}\right\rangle_{R}=\delta_{i j}, \quad\left\langle\phi_{i}, \phi_{j}\right\rangle_{\Omega}=\lambda_{i} \delta_{i j}
$$

5. The $\phi_{i}(\Omega, \Lambda)$ are eigenvectors of the $D$-dimensional DFT, with $\Omega$ and $\Lambda$ interchanged.

$$
T_{\Lambda} F \phi_{i}(\Omega, \Lambda)=\hat{\phi}_{i}(\Lambda, \Omega)
$$

where $F$ is the $D$-dimensional DFT matrix.

6. Consider the norms corresponding to property $4\|\cdot\|_{R}$ and $\|\cdot\|_{\Omega}$. Then among all multidimensional sequences of size $N_{R}$ with frequency support in $P_{\Lambda}, \phi_{1}$ is the one most concentrated in $P_{\Omega}$ with concentration $\left\langle\phi_{1}, \phi_{1}\right\rangle_{\Omega} /\left\langle\phi_{1}, \phi_{1}\right\rangle_{R}=\lambda_{1}$. Similarly, among the sequences of equal frequency support orthogonal to $\phi_{1}, \phi_{2}$ is the most concentrated in $\Omega$.

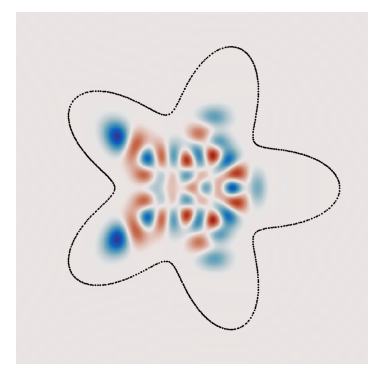

(a) $\lambda_{60} \sim 1-10^{-10}$

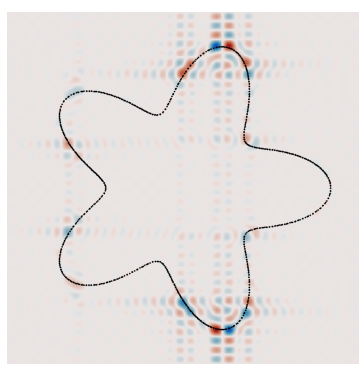

(b) $\lambda_{557} \sim 0.54$

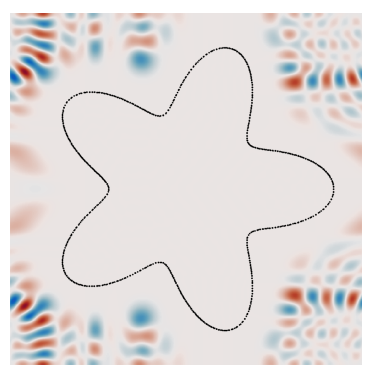

(c) $\lambda_{1300} \sim 10^{-10}$

Figure 4: Fourier series corresponding to periodic discrete prolate spheroidal wave sequences $\phi_{i}$ for different values of the eigenvalue $\lambda_{i}$.

Let us interpret these properties and make the connection to the algorithm. Recall that the matrix $A$ has a particular singular value structure. The left and right singular vectors of $A$ are the eigenvectors of $A A^{\prime}$ and of $A^{\prime} A$ respectively, i.e. they are $\hat{\phi}_{i}(\Omega, \Lambda)$ and $\phi_{i}(\Lambda, \Omega)$, with singular values $\sqrt{\lambda_{i}}$. The singular vectors are, by construction, the periodic discrete prolate spheroidal wave sequences. The 
vectors can be seen as coefficients of a Fourier series and some of the corresponding functions are shown in fig. 4 .

The maximal ratio $\lambda_{1}=\left\|\phi_{1}\right\|_{\Lambda} /\left\|\phi_{1}\right\|_{R}$ with $\lambda_{1} \approx 1$ means that $\phi_{1}$ is almost entirely supported on $\Lambda$ - this in spite of being compactly supported in the (discrete) frequency domain. They are, after all, a finite Fourier series. Such a function is shown in the left panel of fig. 4 . In contrast, the functions corresponding to small eigenvalues are almost entirely supported on the exterior domain $R-\Omega$, as shown in the right panel of the figure. Finally, the middle functions with eigenvalues in the plunge region are supported everywhere. This is illustrated in the middle panel. In particular, these functions are the only ones that are non-neglible in a neighbourhood of the boundary. This is a clear indication that the plunge region is a phenomenon that relates to the boundary of the domain at hand.

The solution to $A x=B$ using a truncated Singular Value Decomposition can be expressed in terms of these generalized discrete Prolate Spheroidal sequences,

$$
\mathbf{a}=\sum_{i=1}^{i_{\max }} \frac{1}{\sqrt{\lambda_{i}}} \hat{\phi}_{i}(\Lambda, \Omega)\left\langle f, \hat{\phi}_{i}(\Omega, \Lambda)\right\rangle
$$

where $i_{\max }$ is determined by the truncation parameter $\epsilon$ and is such that $\lambda_{i_{\max }} \geq \epsilon>\lambda_{i_{\max }+1}$. This expression, combined with fig. 4, clearly illustrates the different steps in Algorithm 1. This is shown further in fig. 5 .

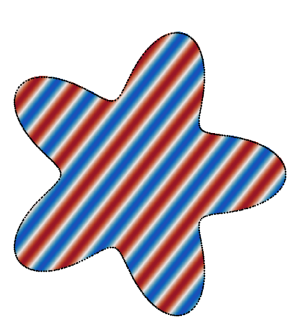

(a) $b$

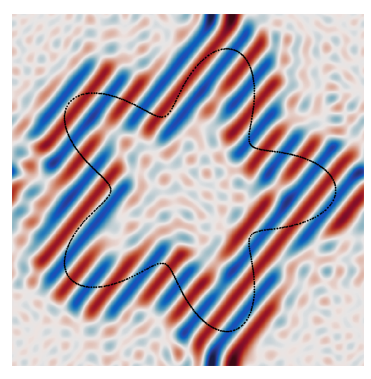

(b) $A y, y=V_{\chi} \Sigma_{\chi}^{-1} U_{\chi}^{\prime} b$

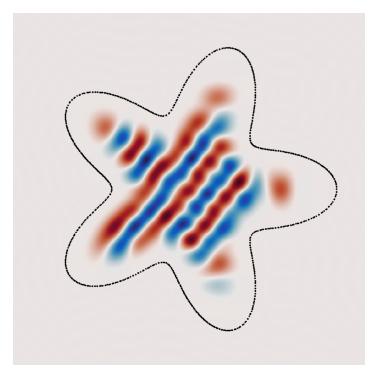

(c) $A z, z=A^{\prime}(b-A y)$

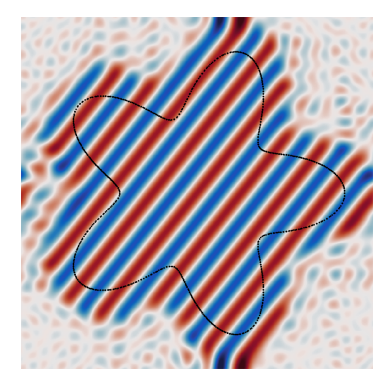

(d) $A(y+z)$

Figure 5: Steps in algorithm 1: Data is given on $\Omega$ (fig. 5a), approximated using the eigenvalues $1-\epsilon>\lambda_{i}>\epsilon$, and yields a good approximation on the boundary (fig. 5b). This solution subtracted from the data (fig. 5c) is easily approximated by a regular Fourier series on the bounding box (fig. $5 \mathrm{~d}$ ).

The vector $y$ found after the first step is based on the middle singular values, which correspond to functions that are supported along the boundary of the domain. The Fourier series with $y$ as its coefficients is shown in fig. 5b: it approximates the data well in a neighbourhood of the boundary. Subtracting this approximation from the original function (as in $b-A y$ ) yields a function that vanishes smoothly towards the boundary of $\Omega$. Hence, this function can be extended by zero and approximated efficiently with a Fourier transform, and that is expressed by the step $z=A^{\prime}(b-A y)$. The vector $z$ is a linear combination of the prolates that are concentrated in the interior of the domain. It is now also clear what the null space of $A$ corresponds to: it consists of linear combinations of the prolates concentrated in the exterior of the domain. Any such prolate can be added to our solution but it will only affect the extension, not the approximation on $\Omega$ itself, unless it is multiplied with a very large coefficient.

\subsection{Singular value profile for generalized discrete Prolate Spheroidal Se- quences}

Proving asymptotic complexity of algorithm 1 needs a bound on $\eta\left(\epsilon, N_{R}\right)$ as $N_{\Lambda}$ increases. As in $[17,36]$, this can be inferred from trace iterates of the operator $T_{\Omega} B_{\Lambda} T_{\Omega}$. After bounding the difference 
between $\operatorname{tr}\left(T_{\Omega} B_{\Lambda} T_{\Omega}\right)$ and $\operatorname{tr}\left(\left(T_{\Omega} B_{\Lambda} T_{\Omega}\right)^{2}\right)$, this bound is shown to be of the same order as $\eta\left(\epsilon, N_{R}\right)$. We formulate our final result in Theorem 9 .

Our bound hinges on two observations:

- The contribution of a single point in $P_{\Omega}$ to $\operatorname{tr}\left(T_{\Omega} B_{\Lambda} T_{\Omega}\right)-\operatorname{tr}\left(\left(T_{\Omega} B_{\Lambda} T_{\Omega}\right)^{2}\right)$ is inversely proportional to the distance between that point and the domain boundary.

- The number of points at a certain distance from the boundary is bounded by the number of boundary points and some terms depending only on domain geometry.

The next section contains a concise illustrated proof of the second observation in the two-dimensional case. The first observation is proven in Section 3.4.2. Due to the discrete nature of the problem, we use some concepts known in digital topology [20].

\subsubsection{Distance away from the boundary for general 2D domains}

For reasons that will become clear later, the metric of choice is the $l_{\infty}$ distance,

$$
d(\mathbf{k}, \mathbf{l})=\|\mathbf{k}-\mathbf{l}\|_{\infty} .
$$

A point $\mathbf{k}$ on a regular grid in two dimensions can have up to 8 neighbors at a distance 1 . We also assign to each point $\mathbf{k}$ a $L_{\infty}$ distance to the boundary of a point set $P$ (or rather to its exterior),

$$
d(\mathbf{k} ;)=\min _{\mathbf{l} \notin P}\|\mathbf{l}-k\|_{\infty} .
$$

Evidently it is true that $\forall \mathbf{k} \notin P: d(\mathbf{k} ; P)=0$, and

$$
\forall \mathbf{k} \in P: d(\mathbf{k} ; P)=\left(\min _{\mathbf{l}: d(\mathbf{l}, \mathbf{k})=1} d(\mathbf{l} ; P)\right)+1 .
$$

Next, let $S_{i}$ denote the points in set $S$ that are a distance $i$ away from the boundary,

$$
S_{i}=\{\mathbf{k} \in S: d(\mathbf{k} ; P)=i\} .
$$

The main result of this section is a bound on the size of these sets, in particular of $\left|S_{i+1}\right|$ in terms of $\left|S_{i}\right|$, which can be obtained using results from digital topology.

Let $\bar{S}_{i}$ denote the points in set $S_{i}$ that have no neighbour in $S_{i+1}$

$$
\bar{S}_{i}=\left\{\mathbf{k} \in S_{i}: \max _{\mathbf{1}: d(\mathbf{1}, \mathbf{k})=1} d(\mathbf{1} ; S) \leq i\right\} .
$$

These definitions are illustrated in Figure 6. For example, in the left panel the solid black dots not connected by a line belong to $\bar{S}_{1}$ : their neighbours are either also in $S_{1}$ or in the exterior of the domain. The black dots connected by a line make up $S_{1} \backslash \bar{S}_{1}$.

Following the terminology of [20], we define a line cell as an adjacent pair of points $(\mathbf{k}, \mathbf{l}): d(\mathbf{k}, \mathbf{l})=1$ and a surface cell as a set of four points where all pairwise distances are 1 . We say that a pair of surface cells is point connected if they share a point, and line-connected if they share two points.

This allows us to state the definition of a regular digital manifold.

Definition 1. [20, Definition 5.14] A point set $S$ on a rectangular grid is a regular digital manifold if

- all points belong to a surface cell;

- for any pair of surface cells there is a line-connected path between them.

We also define a slightly broader class of digital manifolds:

Definition 2. A set $S$ is a pseudoregular digital manifold if 


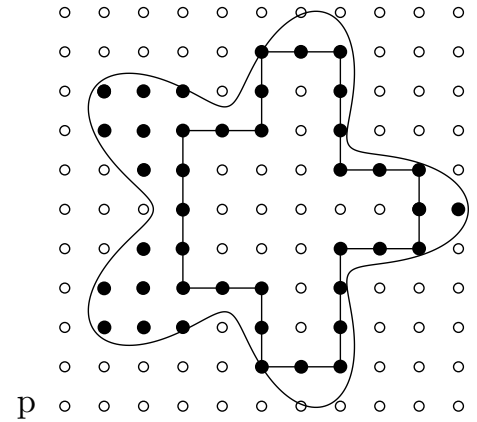

(a) $S_{1}, S_{1} \backslash \overline{S_{1}}$ (regular curve)

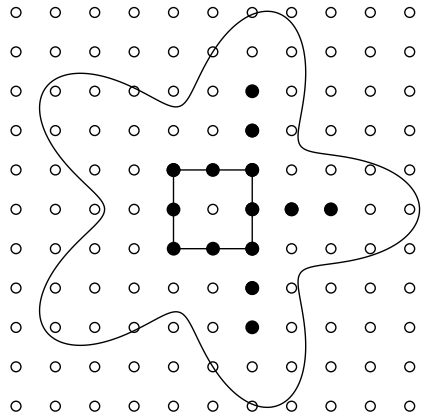

(b) $S_{2}, S_{2} \backslash \bar{S}_{2}$ (regular curve)

Figure 6: An illustration of the sets $S_{1}, \bar{S}_{1}$ and their difference $S_{1} \backslash \bar{S}_{1}$ in a component without holes (left panel), and similarly for $S_{2}$. It is clear that $\left|S_{1}\right| \geq\left|S_{1} \backslash \bar{S}_{1}\right|>\left|S_{2}\right| \geq\left|S_{2} \backslash \overline{S_{2}}\right|$. The set $S_{3}$ in this example consists of a single point.

- all points belong to a surface cell;

- for any pair of surface cells there is a point-connected path between them.

We have the following theorem.

Theorem 1. For any finite 2-dimensional set $S, S-\bar{S}_{1}$ is a finite union of pseudoregular digital manifolds.

Proof. If $S-\bar{S}_{1}$ is empty, then the result is true. Henceforth we assume it is not empty. To prove the first requirement of a pseudoregular manifold, note that because of eq. (33) and eq. (35), every $\mathbf{k} \in S_{2}$ is surrounded by points in $S-\bar{S}_{1}$ and is therefore part of 4 surface cells. Furthermore, because of eq. (35) every point in $S_{1}-\bar{S}_{1}$ has at least one neighbor in $S_{2}$, and is therefore part of a surface cell. Grouping surface cells by point-connectedness, the result is a union of pseudoregular manifolds.

Theorem 2. The distance to the boundary is preserved for all points after the removal of $\bar{S}_{1}$,

$$
\forall \mathbf{k} \in S \backslash \bar{S}_{1}: d\left(\mathbf{k} ; S-\bar{S}_{1}\right)=d(\mathbf{k} ; S) .
$$

Proof. First note that the distance of a point is the minimum over all the 8 connected neighbours plus one. Therefore if $S_{i}$ stays the same, $S_{i+1}$ stays the same. Then note that all neighbors of points in $S_{2}$ are retained in $S-\bar{S}_{1}$.

Theorem 3. [20, Theorem 5.4] The boundary $\delta S$ of a regular 2-dimensional manifold $S$ is itself regular, and the union of closed regular curves.

Theorem 4. [20, Lemma 9.1] A closed 2-dimensional digital curve has 4 more convex corners than non-convex corners.

The combination of these two theorems leads to

Theorem 5. For a 2-dimensional pseudoregular manifold

$$
\left|S_{i+1}\right| \leq\left|S_{i}\right|-4, \quad i \geq 1
$$

Proof. Consider a regular manifold $S$. All points in $S_{1}$ form a closed digital curve with 4 more convex corners than non-convex corners. As illustrated in fig. 7, a point on a straight segment maps to one element of $S_{2}$, a convex corner maps three points of $S_{1}$ to one of $S_{2}$, and a non-convex corner maps one 
points of $S_{1}$ to three of $S_{2}$. Since the points that are being mapped to can also coincide, the bound eq. (37) is obtained.

For a pseudoregular manifold, note that a pair of point connected components can be regarded as 2 regular manifolds, with one point in common. Combining the bounds for both regular manifolds and subtracting the one point in common we end up with eq. (37). Then the theorem can be applied recursively by removing $\bar{S}_{i+1}$, obtaining another pseudoregular set.

For an illustration of this proof, see fig. 7 .

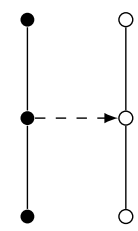

(a) straight

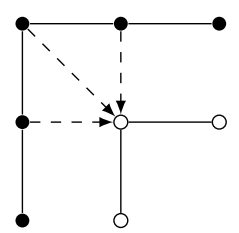

(b) convex

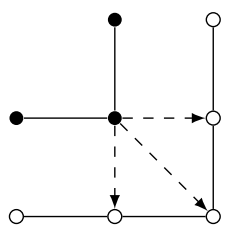

(c) non-convex

Figure 7: Illustration accompanying theorem 5.

We conclude with a generalization that allows for a finite number of holes in a set. The set $S_{i+1}$ may be larger in this case than $S_{i}$, but the small growth does not invalidate the asymptotic complexity in the next section.

Theorem 6. For a 2-dimensional set containing c 8-connected components and h holes, the number of points a distance $i$ away from the boundary is bounded by

$$
\left|S_{i+1}\right| \leq\left|S_{i}\right|-4(c-h), \quad i \geq 1 .
$$

Proof. For $c$ 8-connected components, theorem 5 holds individually for each $S_{i j}$. Thus the bound for the combined sets $S_{i}$ is

$$
\left|S_{i+1}\right| \leq\left|S_{i}\right|-4 c .
$$

A hole in this context is a connected component not in $P$ but entirely surrounded by it. Denote by $S_{i B}$ the points whose closest neighbor not in $P$ is in the hole. Then a similar reasoning to theorem 5 shows that

$$
\left|S_{i, B}\right| \leq\left|S_{i} \backslash \bar{S}_{i, B}\right|<\left|S_{i+1, B}\right|+4 .
$$

Summing the bounds completes the proof.

Remark 2. Theorem 5 does not hold in three dimensions and higher. In fact, the set $S_{i+1}$ can be larger than $S_{i}$ even for domains without a hole. A domain with an intrusion can have interior non-convex angles, at which a single point in $S_{i}$ maps to many points in $S_{i+1}$.

\subsubsection{Bounding $\eta\left(\epsilon, N_{R}\right)$}

Theorem 7. Let $T_{\Omega}$ and $B_{\Lambda}$ be as in eqs. (26) and (27). We are interested in the behavior for large $n_{\Lambda}$, with constant oversampling $\gamma=n_{\Lambda} / P_{\tilde{R}}$. Furthermore, let $N_{\delta \Omega}\left(n_{\Lambda}\right)$ denote the number of points in $P_{\delta \Omega}$ neighbouring the boundary, i.e. $S_{1}$ from the previous section:

$$
P_{\delta \Omega}=\left\{\mathbf{k} \in \Omega \quad \mid \quad \exists \mathbf{l},\|\mathbf{l}\|_{\infty}=1: \mathbf{k}+\mathbf{l} \notin \Omega\right\} .
$$

We further assume that the limit

$$
\lim _{n_{\Lambda} \rightarrow \infty}\left(h\left(P_{\Omega}\right)-c\left(P_{\Omega}\right)\right)=C
$$


exists with $C<\infty$, where $h\left(P_{\Omega}\right)$ and $c\left(P_{\Omega}\right)$ are as before the number of holes and distinct connected components of $P_{\Omega}$. Then for the operator $T_{\Omega} B_{\Lambda} T_{\Omega}$

$$
\lim _{n_{\Lambda} \rightarrow \infty} \operatorname{tr}\left(T_{\Omega} B_{\Lambda} T_{\Omega}\right)-\operatorname{tr}\left(T_{\Omega} B_{\Lambda} T_{\Omega}^{2}\right)=O\left(N_{\delta \Omega} \log n_{\Lambda}\right) .
$$

Proof. The trace of $T_{\Omega} B_{\Lambda} T_{\Omega}$ is

$$
\begin{aligned}
\operatorname{tr}\left(T_{\Omega} B_{\Lambda} T_{\Omega}\right) & =\sum_{\mathbf{k} \in I_{\Omega}} B(\mathbf{k}-\mathbf{k}) \\
& =N_{\Omega} B(\mathbf{0})=\frac{N_{\Omega} N_{\Lambda}}{N_{R}} .
\end{aligned}
$$

For the squared operator trace, note that

$$
\operatorname{tr}\left(\left(T_{\Omega} B_{\Lambda} T_{\Omega}\right)^{2}\right)=\left\|T_{\Omega} B_{\Lambda} T_{\Omega}\right\|_{F}=\sum_{\mathbf{k} \in I_{\Omega}} \sum_{\mathbf{l} \in I_{\Omega}}\left|\left(T_{\Omega} B_{\Lambda} T_{\Omega}\right)_{\mathbf{k}, 1}\right|^{2}
$$

Now, define an intermediate function

$$
f(\mathbf{k})=\sum_{\mathbf{l} \in I_{\Omega}}\left|\left(T_{\Omega} B_{\Lambda} T_{\Omega}\right)_{\mathbf{k}, \mathbf{l}}\right|^{2}, \quad \operatorname{tr}\left(\left(T_{\Omega} B_{\Lambda} T_{\Omega}\right)^{2}\right)=\sum_{\mathbf{k} \in I_{\Omega}} f(\mathbf{k}) .
$$

This $f$ can be rewritten as

$$
\begin{aligned}
f(\mathbf{k}) & =\sum_{\mathbf{k} \in I_{\Omega}}|B(\mathbf{k}-\mathbf{l})|^{2} \\
& =\sum_{\mathbf{l} \in I_{R}}|B(\mathbf{k}-\mathbf{l})|^{2}-\sum_{\mathbf{l} \in\left(I_{R} \backslash I_{\Omega}\right)}|B(\mathbf{k}-\mathbf{l})|^{2}
\end{aligned}
$$

The first sum is equal to $\frac{N_{\Lambda}}{N_{R}}$ through Parseval's equation. The second term is the sum over the index set $I_{R} \backslash I_{\Omega}$. As a shorthand notation, use

$$
q_{\mathbf{k}}=d\left(\mathbf{k} ; I_{\Omega}\right) .
$$

The largest inscribed square around $\mathbf{k}$ is then given by $\mathbf{k}+Q_{\mathbf{k}} \times Q_{\mathbf{k}}$, see fig. 8. with $Q_{\mathbf{k}}=\left\{-q_{\mathbf{k}}+\right.$ $\left.1, \ldots, q_{\mathbf{k}}-1\right\}$. Restricting $I_{\Omega}$ to this square and using that due to periodicity $\sum_{\mathbf{l} \in I_{R}}|B(\mathbf{l})|^{2}=$ $\sum_{\mathbf{l} \in I_{R}-\mathbf{k}}|B(\mathbf{l})|^{2}$, the last sum can be bounded by

$$
\begin{aligned}
\sum_{\mathbf{l} \in\left(I_{R} \backslash I_{\Omega}\right)}|B(\mathbf{k}-\mathbf{l})|^{2} & <\sum_{\mathbf{l} \in\left(I_{R} \backslash\left(\mathbf{k}+Q_{\mathbf{k}} \times Q_{\mathbf{k}}\right)\right.}|B(\mathbf{k}-\mathbf{l})|^{2} \\
& =\sum_{\mathbf{l} \in\left(I_{R} \backslash\left(Q_{\mathbf{k}} \times Q_{\mathbf{k}}\right)\right.}|B(\mathbf{l})|^{2} \\
& =\left(\sum_{k \in R_{d} \backslash Q_{\mathbf{k}}}|B(k)|_{d}^{2}\right)^{2}+2\left(\sum_{k \in R_{d} \backslash Q_{\mathbf{k}}}|B(k)|^{2} \sum_{k \in Q_{\mathbf{k}}}|B(k)|^{2}\right) .
\end{aligned}
$$

Here $R_{d}=\left\{0, \ldots, n_{R}-1\right\}$, and $B(k)$ is the one-dimensional kernel.

From [36], the first sum can be bounded by

$$
\sum_{k \in R_{d} \backslash Q_{\mathbf{k}}}|B(k)|_{d}^{2}=\sum_{k=q_{\mathbf{k}}}^{n_{R}-q_{\mathbf{k}}}\left(\frac{\sin (\pi k / \gamma)}{n_{R} \sin \left(\pi k / n_{R}\right)}\right)^{2}<\frac{1}{4 q_{\mathbf{k}}}+\frac{\gamma}{16 q_{\mathbf{k}}^{2}},
$$




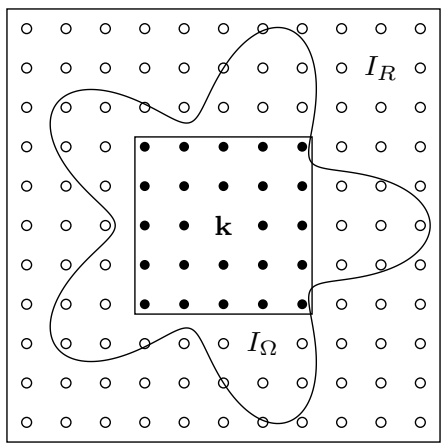

Figure 8: The largest inscribed square in $I_{\Omega}$ around any point $\mathbf{k}$ is $\mathbf{k}+Q_{\mathbf{k}} \times Q_{\mathbf{k}}$. In this figure $q_{\mathbf{k}}=3$, leading to a $5 \times 5$ square.

Further, $\sum_{k \in Q_{\mathbf{k}}}|B(k)|_{d}^{2}<\gamma$. Then eq. (49) can be bounded by a rational polynomial in $q_{\mathbf{k}}$.

$$
\sum_{\mathbf{l} \in\left(I_{R} \backslash I_{\Omega}\right)}|B(\mathbf{k}-\mathbf{l})|^{2}<\frac{\gamma}{2} q_{\mathbf{k}}^{-1}+\left(\frac{\gamma^{2}}{2^{3}}+\frac{1}{2^{4}}\right) q_{\mathbf{k}}^{-2}+\frac{\gamma}{2^{5}} q_{\mathbf{k}}^{-3}+\frac{\gamma^{2}}{2^{8}} q_{\mathbf{k}}^{-4},
$$

with all coefficients independent of $N_{R}$. Then

$$
\begin{aligned}
\operatorname{tr}\left((T B T)^{2}\right) & =\sum_{\mathbf{k} \in I_{\Omega}} f(\mathbf{k}) \\
& >\frac{N_{\Omega} N_{\Lambda}}{N_{R}}-\sum_{\mathbf{k} \in I_{\Omega}}\left(\gamma q_{\mathbf{k}}^{-1}+O\left(q_{\mathbf{k}}^{-2}\right)\right) .
\end{aligned}
$$

Now recall from section 3.4.1 that $I_{\Omega}$ can be divided into sets $S_{i}=\left\{\mathbf{k}: q_{\mathbf{k}}=i\right\}$. Theorem 6 states that $\left|S_{i+1}\right|<\left|S_{i}\right|-4(c-h)$. Furthermore, the size of the bounding box dictates that $q_{\mathbf{k}}$ can never exceed $\frac{n_{R}}{2}$. With this in mind it is easier to sum over the regions $S_{i}$ than over all points at once. This leads to a bound

$$
\begin{aligned}
\frac{N_{\Omega} N_{\Lambda}}{N_{R}}-\operatorname{tr}\left((T B T)^{2}\right) & <\sum_{i=1}^{n_{R} / 2} \sum_{S_{i}}\left(\gamma i^{-1}+O\left(i^{-2}\right)\right) \\
& <\sum_{i=1}^{n_{R} / 2}\left(N_{\delta \Omega}+4 i(h-c)\right)\left(\gamma i^{-1}+O\left(i^{-2}\right)\right) \\
& <C_{1} N_{\delta \Omega} \log n_{R}+C_{2} N_{\delta \Omega}
\end{aligned}
$$

Equations (44) and (54) combined give the desired result.

Next, we want to relate the difference between iterated traces to the plunge region. This relies on a fairly general counting argument. Recall that the trace of a matrix equals the sum of its eigenvalues, and the trace of a matrix squared equals the sum of the squares of its eigenvalues.

Theorem 8. Let $1>\lambda_{1}(N)>\lambda_{2}(N)>\cdots>\lambda_{N}(N)>0$ be a given ordered series where

$$
\begin{aligned}
\sum_{i=1}^{N} \lambda_{i}(N) & =C N \\
\sum_{i=1}^{N} \lambda_{i}(N)^{2} & =C N-g(N)
\end{aligned}
$$

where $g(N)=o(N)$ is a positive function. Then $\left|\left\{\lambda_{k}: \epsilon<\lambda_{k}<1-\epsilon\right\}\right|=O(g(N))$. 
Proof. Define $k_{\min }$ and $k_{\max }$ as the limits of the intermediate region

$$
k_{\min }=\underset{k}{\arg \min } \lambda_{k}: \lambda_{k}<1-\epsilon, \quad k_{\max }=\underset{k}{\arg \max } \lambda_{k}: \lambda_{k}>\epsilon .
$$

Then $\forall k>k_{\min }: \lambda_{k}^{2}<(1-\epsilon) \lambda_{k}$ and $\forall k \leq k_{\min }: \lambda_{k}^{2}<(1-\epsilon) \lambda_{k}+\epsilon$, so that

$$
\sum_{k} \lambda_{k}^{2}<(1-\epsilon) \sum_{k} \lambda_{k}+\epsilon k_{\min }
$$

Substituting eqs. (55) and (56) leads to

$$
k_{\min }>C N-\frac{g(N)}{\epsilon} .
$$

Similarly, $\forall k<k_{\max }: \lambda_{k}^{2}<(1+\epsilon) \lambda_{k}-\epsilon$ and $\forall k \geq k_{\max }: \lambda_{k}^{2}<(1+\epsilon) \lambda_{k}$, so that

$$
\sum_{k} \lambda_{k}^{2}<(1+\epsilon) \sum_{k} \lambda_{k}-\epsilon k_{\max }
$$

Combined with eqs. (55) and (56) this yields the upper bound

$$
k_{\max }<C N+\frac{g(N)}{\epsilon}
$$

Theorem 9. Let $\Omega, T_{\Omega}, B_{\Lambda}$ and $P_{\delta \Omega}$ be as in Theorem $\%$. Then for the operator $T_{P_{\Omega}} B_{\Lambda} T_{P_{\Omega}}$

$$
\eta\left(\epsilon, N_{R}\right)=O\left(N_{\delta \Omega} \log n_{R}\right)
$$

where $\eta\left(\epsilon, N_{R}\right)$ is as in eq. (11).

Proof. The proof follows directly from theorem 7 and theorem 8, and noting that for square matrices $\operatorname{Tr}\left(A^{k}\right)=\sum_{k} \lambda^{k}$.

Remark 3. Theorem 9 gives a bound in terms of $N_{\delta \Omega}\left(n_{\Lambda}\right)$. For any 2-dimensional non-fractal domain, $N_{\delta \Omega}=\mathcal{O}\left(n_{\Lambda}\right)$. To see this, note that $d=\lim _{n_{\Lambda} \rightarrow \infty} \frac{\log N_{\delta \Omega}}{\log n_{\Lambda}}$ is equal to the box-counting or MinkowskiBouligand definition of the boundary dimension [13]. For a non-fractal domain this is equal to the topological dimension of the boundary which is 1 .

Remark 4. As mentioned in the introduction, Theorem 9 leads to an $O\left(N_{\Lambda}^{2} \log \left(N_{\Lambda}\right)^{2}\right)$ complexity for algorithm 1 on $2 D$ domains. It is however difficult to extend the results from section 3.4.1 to higher dimensions, as there is no straightforward equivalent of theorem 5. If extended to those domains theorem 9 yields asymptotic reductions in higher dimensions, though the savings have diminishing returns, generally of the order $O\left(N^{3 d-2}\right)$ versus $O\left(N^{3 d}\right)$ for a full $S V D$.

\section{Numerical results}

This section contains examples and numerical results for various two-dimensional domains and method parameters. The aim is to demonstrate the asymptotic complexity, convergence properties and robustness of the algorithm. Algorithm 1 was implemented using a randomized algorithm for the low rank subsystem. A selection of possible geometries was used, shown in fig. 9. All domains are normalized to have equal area. These domains where chosen to showcase differences in results for contrasting properties:

- The square and diamond show the method is not rotation invariant. 
- The square and disk show the effect of corners

- The disk and ring show the effect of a simply connected domain versus a not simply connected domain.

- A double asteroid is included to study boundaries that are not smooth.

The precise effect of the domain on complexity and accuracy is discussed in section 4.3.

Throughout these experiments, the basis of choice is a Fourier basis on the rectangle $[-T, T] \times$ $[-T, T]$ with $N^{2}$ degrees of freedom. Unless specified otherwise, the value for $T$ is 2 and the oversampling factor $N_{\Omega} / N_{\Lambda}$ is taken to be 4 . The cutoff $\epsilon$ (expressed through the estimate of $\eta\left(\epsilon, N_{R}\right)$ ) is consistently $10^{-14}$.
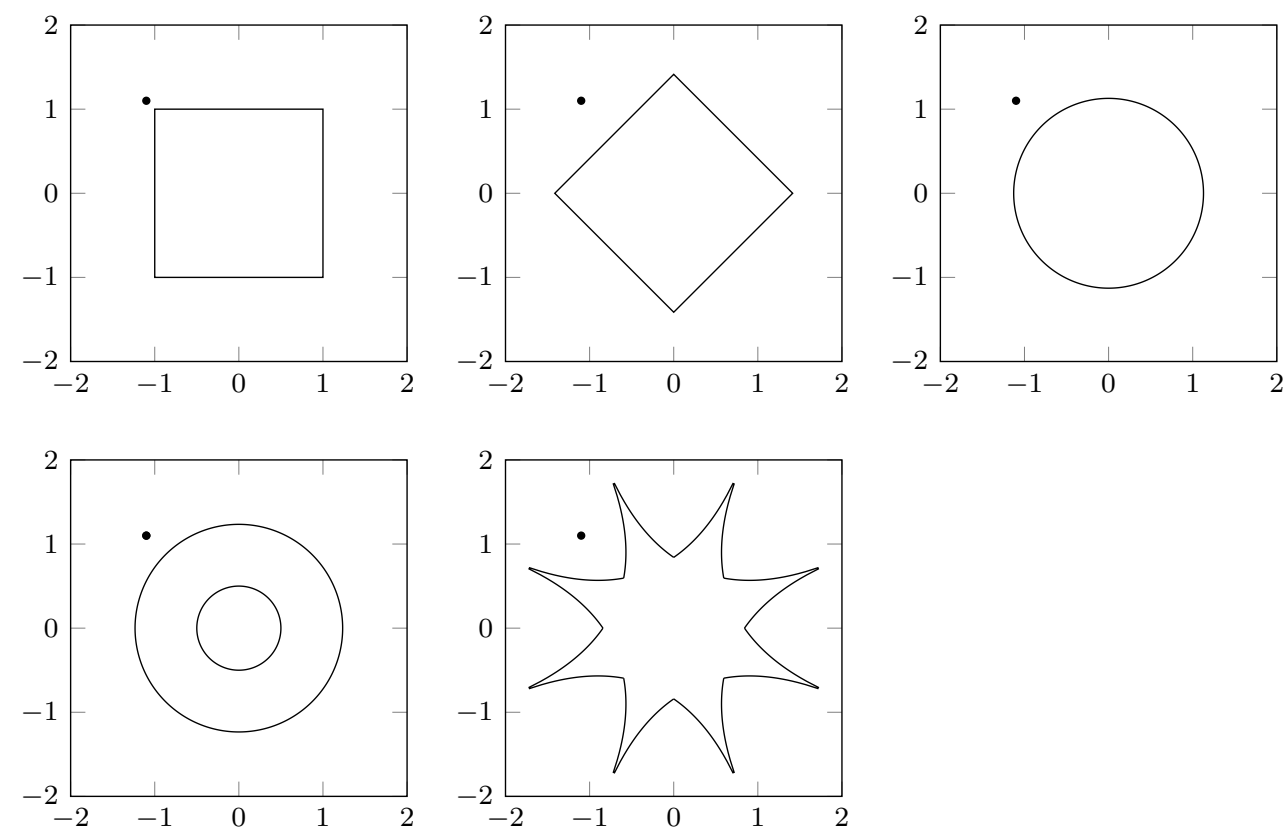

Figure 9: Test domains used throughout this section. The dot marks the location of the singularity in the third test function.

Remark 5. Faster options exist to approximate functions on rectangular regions, including tensor product 1D Fourier Extensions. The experiments in this section do not exploit this structure.

\subsection{Complexity}

Figure 10 shows execution time for Fourier frame approximations with increasing degrees of freedom. The approximant is irrelevant here since complexity of algorithm 1 is independent of the right hand side. Our algorithm computes the equivalent of a truncated SVD and is applied to the sampled function in a single step. The domain is also largely irrelevant since the plunge region size is similar for the chosen domains, see section 4.3. Therefore the timings are shown for just the one example: approximating

$$
f(x, y)=e^{(x+y)} \cos (20 x y)
$$

on a disk of area 4 .

The results confirm the $O\left(N_{\Lambda}^{2} \log ^{2} N_{\Lambda}\right)$ complexity of the projection algorithm, with the dominant cost being the SVD used in the randomized SVD solver. Unfortunately, the direct method is only 


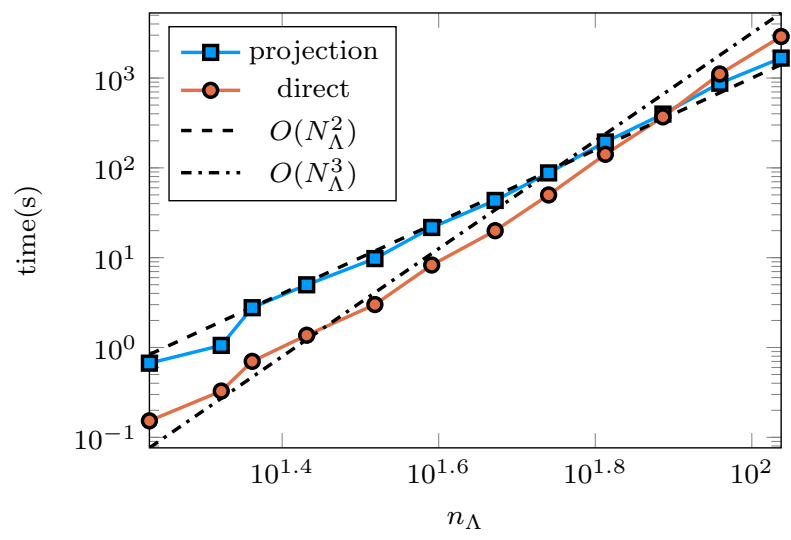

Figure 10: Execution time for a 2D frame approximation, using both a direct solver and the projection algorithm. $O\left(N_{\Lambda}^{2}\right)$ and $O\left(N_{\Lambda}^{3}\right)$ shown dashed in black.

overtaken for $n_{\Lambda}>90$, making the projection method mostly suited for problems requiring a large number of degrees of freedom, such as oscillatory functions. However, the algorithm provides control over the regularization through the chosen accuracy $\epsilon$ of the low rank problem, which the direct solver lacks. In the presence of noise on the order of $\delta$, one may want to choose $\epsilon>\delta$ in order to retain numerical stability [3].

\section{$4.2 \quad$ Accuracy}

To show convergence, the frame approximation method was applied to a set of test functions, for increasing degrees of freedom. The test functions are

- A well-behaved, smooth function

$$
f(x, y)=e^{x+y} .
$$

- A function with a singularity inside the bounding box

$$
f(x, y)=\frac{1}{\left((x-1.1)^{2}+(y-1.1)^{2}\right)^{2}} .
$$

- An oscillatory function

$$
f(x, y)=\cos (24 x-32 y) \sin (21 x-28 y) .
$$

- A function with a discontinuity in the first order partial derivatives

$$
f(x, y)=|x y| .
$$

The results are shown in figs. 11 and 12 , for the residual norm $\|A x-b\|_{2}$ on the one hand and for the largest point error $\|\mathcal{F}-f\|_{\infty}$ on the other hand, sampled randomly in the domain (10000 samples). There are a few interesting observations to be made regarding the convergence behavior for different target functions.

- The approximation error for the smooth function shows superalgebraic convergence on all domains, strengthening claims in this regard [3]. The only exception is the star-shaped point error.

- The approximation error for the oscillatory function behaves exactly as expected, decreasing rapidly once the highest oscillatory mode can be resolved by the basis functions. 
- Figure 12b shows the results for a function with a singularity right outside the domain of interest. Similar to the 1D case, this results in a slower, yet still superalgebraic rate of convergence.

- A function that has $s$ continuous derivatives will exhibit order $s+1$ convergence, as seen in fig. $12 \mathrm{~d}$, for the residual error. The largest point error shows very little, if any, convergence..

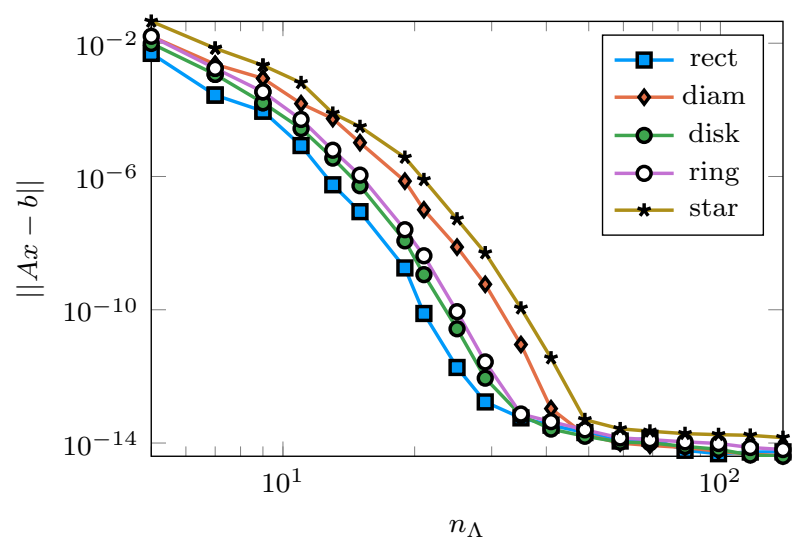

(a) $f(x, y)=e^{x+y}$

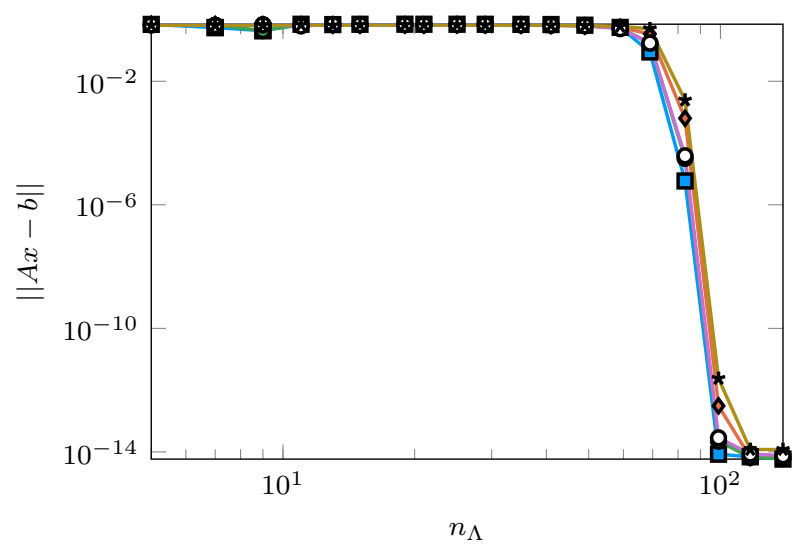

(c) $f(x, y)=\cos (24 x-32 y) \sin (21 x-28 y)$

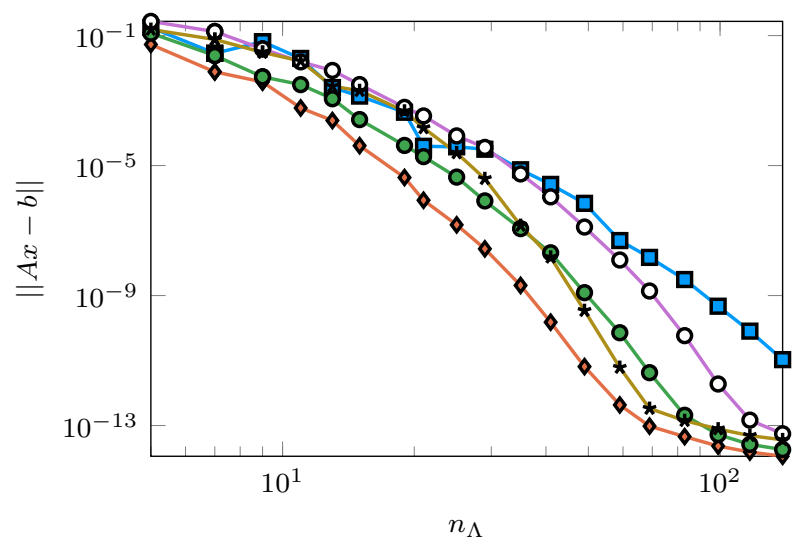

(b) $f(x, y)=\frac{1}{\left((x-1.1)^{2}+(y-1.1)^{2}\right)^{2}}$

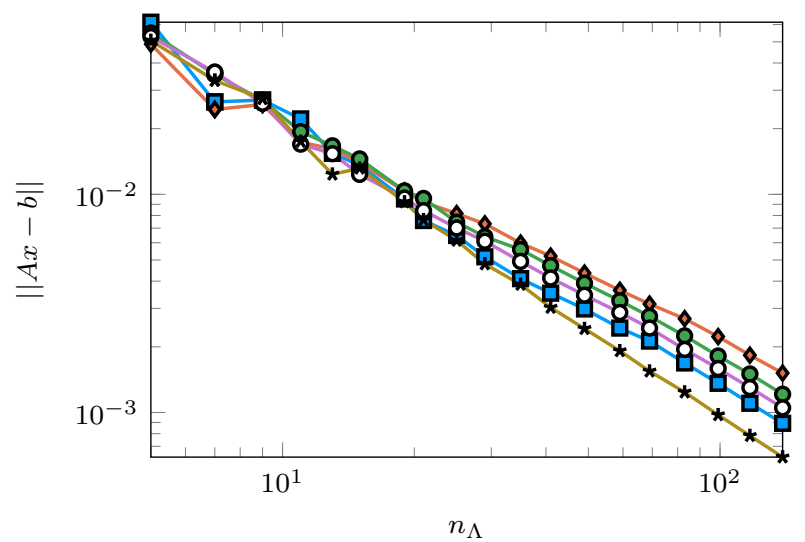

(d) $f(x, y)=|x y|$

Figure 11: Residuals for the approximations from fig. 12.

Remark 6. Our algorithm only ensures small $\|A x-b\|$ if a suitable $x$ exists. The effect of sampling on $\|\mathcal{F}-f\|$ will be studied in a follow-up paper on [3] focusing on the discrete case, and commented on in the next section.

\subsection{Influence of domain shape}

\subsubsection{Plunge region estimates}

Theorem 9 leads to an estimate of the plunge region of the form

$$
\eta\left(\epsilon, N_{R}\right)=C_{1} N_{\delta \Omega} \log n_{R}+O\left(N_{\delta \Omega}\right)
$$




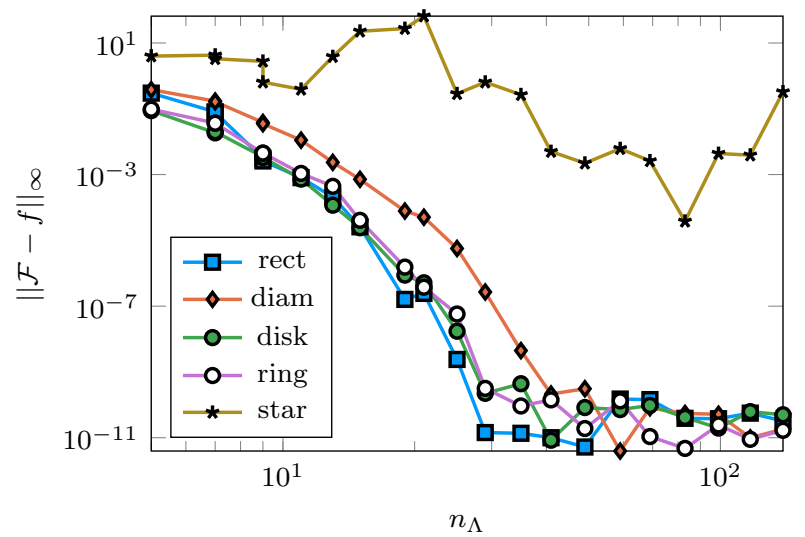

(a) $f(x, y)=e^{x+y}$

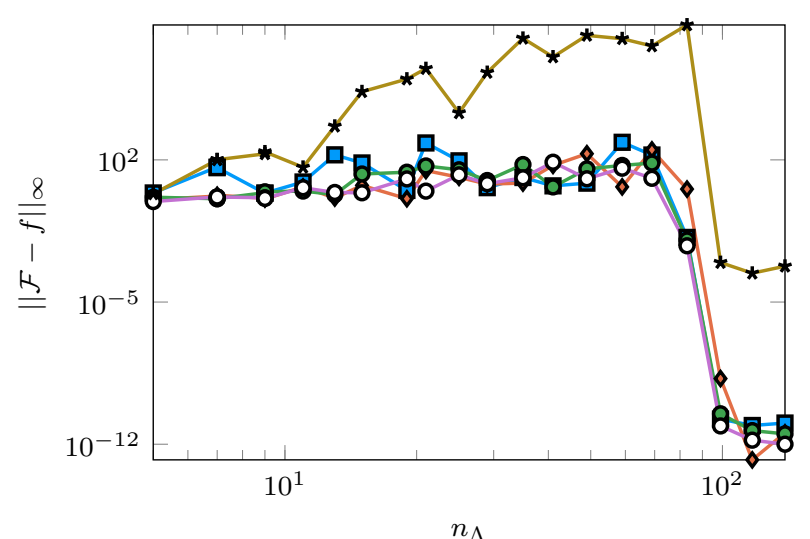

(c) $f(x, y)=\cos (24 x-32 y) \sin (21 x-28 y)$

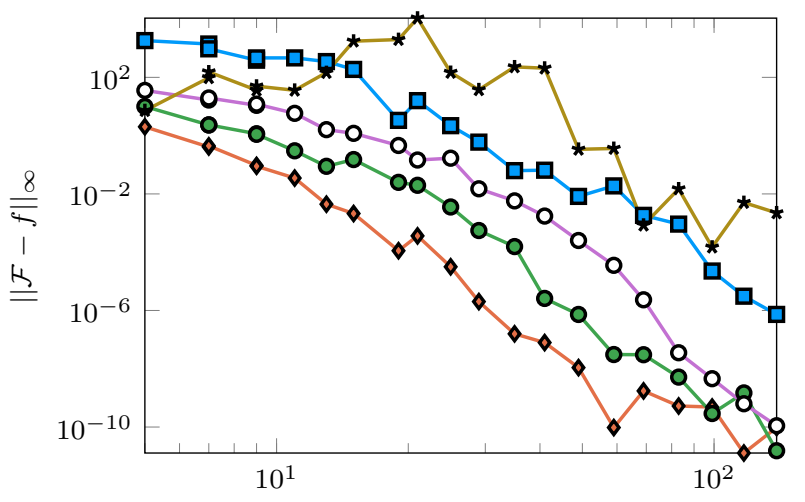

(b) $f(x, y)=\frac{1}{\left((x-1.1)^{2}+(y-1.1)^{2}\right)^{2}}$

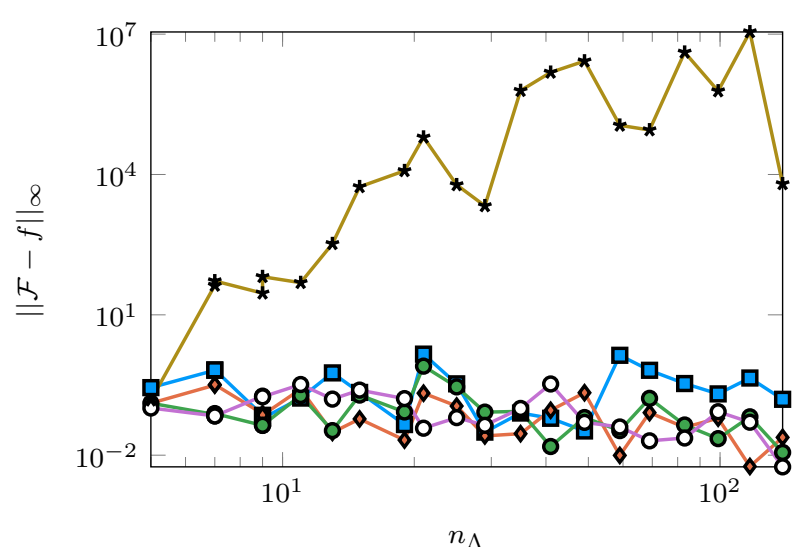

(d) $f(x, y)=|x y|$

Figure 12: Maximum pointwise error for a 2D frame approximation, for different domains and approximants. 
where $C_{1}=\left(\frac{n_{\Lambda}}{n_{R}}\right)^{D-1} \frac{1}{\epsilon^{2}}$. This is because the eigenvalues $\lambda_{i}$ of $T_{\Omega} B_{\Lambda} T_{\Omega}$ are the squares of the singular values $\sigma_{i}$ of the collocation matrix $A$, so that

$$
\epsilon<\sigma_{i}<1-\epsilon \Leftrightarrow \epsilon^{2}<\lambda_{i}<1-\epsilon+\epsilon^{2}
$$

The constant $C_{1}$ is a gross overestimate, as shown in fig. 13, which plots the ratio $\eta\left(\epsilon, N_{R}\right) /\left(\sqrt{n_{R}} \log n_{R}\right)$ as a function of $N_{\Lambda}$. The ellipse, square and diamond seem to reach the asymptotic behavior eq. (63)

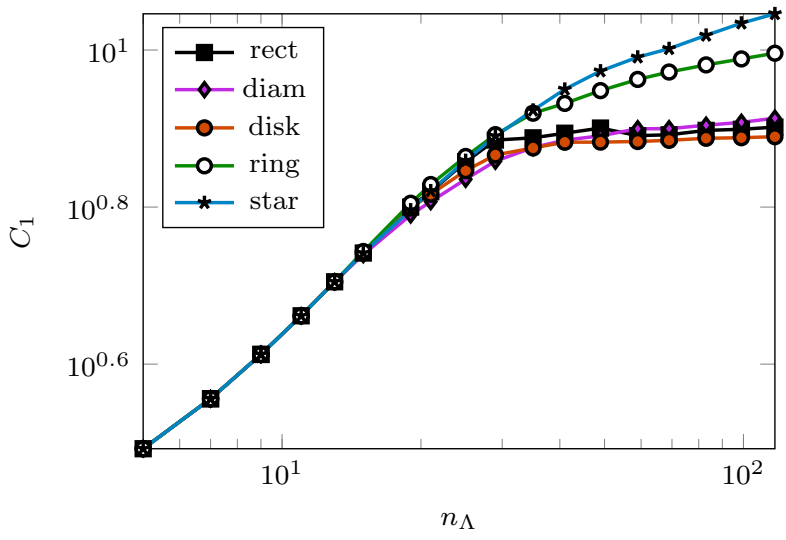

Figure 13: Estimate of plunge region size with respect to $N_{\delta \Omega} \log n_{R}$.

with a constant $C_{1} \sim 10$. The ring and star domain have not yet reached their plateau, but it is reasonable to assume this plateau, like the bound from theorem 9 , is proportional in some way to the domain boundary length. Using the Euclidean length, the plateau for the ring would be at $10^{1.08}$ and for the star at $10^{1.39}$, both plausible from fig. 13 .

Remark 7. When using an $\epsilon$ close to machine precision, an alternative to using an estimate for the plunge region rank is to use an adaptive form of the random matrix algorithm for unknown ranks. As per [21], this eliminates the need for a difficult estimation of $C_{1}$, at a maximum factor 2 increase in cost.

\subsubsection{Influence on convergence}

The influence of domain shape on convergence is readily apparent from figs. 12a to 12d. There are a number of factors that combined lead to the differences seen between the domains.

The maximum pointwise error In fig. 12 the error was taken as the infinity norm over $\Omega$ for $F-f$, calculated over 10000 random samples of $\Omega$. However, the actual approximation $F-f$ in all these experiments was computed from an equispaced grid of collocation points. Some points in $\Omega$, e. g. at the spikes of the star shape may be far away from the equispaced grid. Since no information about these points was taken into account, convergence in these areas cannot be expected until they are sufficiently covered by the grid. This is most apparent in the star-shaped domain, as it has sharp features that are difficult to cover with an equispaced grid. This problem is unique to the higher-dimensional case, as in the one-dimensional problem the endpoints can be guaranteed to be included.

Figure 11 contains the experiments from fig. 12 but now shows the relative error in the grid points only. The results show that all approximations do converge as expected in the collocation points. The difference is, as was expected most apparent in the star-shaped region.

Moreover, this shows that the solution the algorithm provides to the least squares system (9) is almost always accurate up to the supplied tolerance. 
Proximity to the singularity In the $1 \mathrm{D}$ case, the effect of the presence of a singularity on the convergence rate was first quantified in [15] and later expanded upon in [2]. They found that the first regime of convergence is $\|\mathcal{F}-f\|=c \rho^{-N}$ where $\rho$ depends on the minimum of the closest distance to the bounding box and the distance to the singularity.

This behaviour returns in figs. $11 \mathrm{~b}$ and $12 \mathrm{~b}$. With the singularity of the test function

$$
f(x, y)=\frac{1}{\left((x-1.1)^{2}+(y-1.1)^{2}\right)^{2}}
$$

located at $(1.1,1.1)$, the rectangle is closest to the singularity. In rough order of proximity to the singularity, the other domains are star, ring, circle and diamond. The effect is most apparent in fig. 11b, where the convergence rate of the error for the diamond shape is significantly higher than the rate for the rectangle, where for test functions without singularities they differ much less.

\subsection{Robustness}

To ensure the results remain stable for large $N_{\Lambda}$, fig. 14 shows the approximation of a function

$$
f(x, y)=\sin \left(\frac{n_{\Lambda}}{2}(x+y)\right)
$$

for increasing degrees of freedom $N_{\Lambda}$ on a unit circle. This showcases the close relationship between the number of degrees of freedom needed per wavelength and the size of the extension region. For $T=1.2$ and $T=2$, the extension region is narrow enough for the approximation to resolve the oscillation. The main difference here is the convergence rate, which is slower for smaller $T$ as per the $1 \mathrm{D}$ case. For $T=3$, the highest frequency mode present in the Fourier basis of degree $N_{\Lambda}$ is not enough to resolve the function, and it is impossible for convergence to occur.

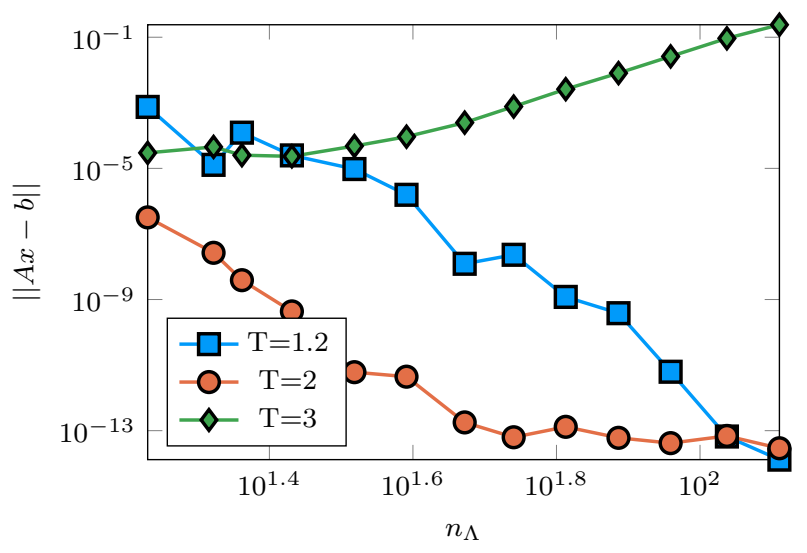

Figure 14: Accuracy for a 2D frame approximation for an increasingly oscillatory function, and different extension regions $R$.

\section{Conclusion}

In this paper we examined an algorithm that provides for Fourier Frame approximations on arbitrary domains. The generality of this approach was demonstrated by approximating a variety of function on several different domains, up to machine precision. The ease of implementing and applying the method, combined with the demonstrated superalgebraic convergence make for an appealing direction in higher dimensional frame approximations. 
Speeding up the algorithm hinges on the occurence of a certain singular value profile. We proved that when using a collocation approach, the matrices exhibit this sort of profile. This newfound result connects the complexity of the algorithm to a certain measure of the domain boundary. This is related to a long history of multidimensional generalizations of Slepian's Prolate Spheroidal Wave functions, albeit in a discrete sense.

The natural decoupling of the problem into a lower-dimensional problem corresponding to the boundary of the domain and a well-conditioned problem in the interior may very well lead to further improvements in complexity.

\section{Acknowledgements}

The authors gratefully acknowledge interesting discussions on the topic of this paper with Ben Adcock, Vincent Coppé, Evren Yarman and Marcus Webb. The authors are supported by FWO Flanders projects G.A004.14 and G.0641.11.

\section{References}

[1] B. Adcock and D. Huybrechs. On the resolution power of Fourier extensions for oscillatory functions. arXiv preprint arXiv:1105.3426, pages 1-36, 2011.

[2] B. Adcock, D. Huybrechs, and J. Martín-Vaquero. On the numerical stability of Fourier extensions. Found. Comp. Math., 14:635-687, 2014.

[3] B. E. N. Adcock and D. Huybrechs. Frames and numerical approximation.

[4] N. Albin and O. P. Bruno. A spectral FC solver for the compressible NavierStokes equations in general domains I: Explicit time-stepping. Journal of Computational Physics, 230(16):6248-6270, 2011.

[5] L. Borcea, G. Papanicolaou, and F. G. Vasquez. Edge illumination and imaging of extended reflectors. SIAM Journal on Imaging Sciences, 1(1):75-114, 2008.

[6] J. P. Boyd. A Comparison of Numerical Algorithms for Fourier Extension of the First, Second, and Third Kinds. Journal of Computational Physics, 178(1):118-160, may 2002.

[7] J. P. Boyd. Fourier embedded domain methods: extending a function defined on an irregular region to a rectangle so that the extension is spatially periodic and C. Applied Mathematics and Computation, 161(2):591-597, 2005.

[8] O. Bruno. Fast, high-order, high-frequency integral methods for computational acoustics and electromagnetics. In Topics in Computational Wave Propagation, volume 31, pages 43-82. Springer, Berlin, 2003.

[9] O. Bruno, Y. Han, and M. Pohlman. Accurate, high-order representation of complex threedimensional surfaces via Fourier continuation analysis. Journal of Computational Physics, 227(2):1094-1125, dec 2007.

[10] O. P. Bruno and M. Lyon. High-order unconditionally stable FC-AD solvers for general smooth domains I. Basic elements. Journal of Computational Physics, 229(6):2009-2033, mar 2010.

[11] A. Bueno-Orovio. Fourier embedded domain methods: Periodic and C??? extension of a function defined on an irregular region to a rectangle via convolution with Gaussian kernels. Applied Mathematics and Computation, 183(2):813-818, 2006. 
[12] R. Duffin and A. Schaeffer. A class of nonharmonic Fourier series. Trans. Amer. Math. Soc., 72:341-366, 1952.

[13] K. Falconer. Fractal Geometry, volume 11. John Wiley \& Sons, Ltd, Chichester, UK, sep 1990.

[14] B. Fornberg and C. Piret. On choosing a radial basis function and a shape parameter when solving a convective PDE on a sphere. Journal of Computational Physics, 227(5):2758-2780, 2008.

[15] D. Huybrechs. On the Fourier extension of nonperiodic functions. SIAM Journal on Numerical Analysis, 47(6):4326-4355, 2010.

[16] A. Jain and S. Ranganath. Extrapolation algorithms for discrete signals with application in spectral estimation. Acoustics, Speech and Signal ..., 29(4):830-845, aug 1981.

[17] H. Landau. On Szegö's eigenvalue distribution theorem and non-Hermitian kernels.

[18] H. Landau and H. Pollak. Prolate spheroidal wave functions, Fourier analysis and uncertainty II. Bell Syst. Tech. J, 1961.

[19] H. Landau and H. Widom. Eigenvalue distribution of time and frequency limiting. Journal of Mathematical Analysis and Applications, 77(2):469-481, 1980.

[20] C. Li. Digital and Discrete Geometry.

[21] E. Liberty, F. Woolfe, P.-G. Martinsson, V. Rokhlin, and M. Tygert. Randomized algorithms for the low-rank approximation of matrices. Proceedings of the National Academy of Sciences of the United States of America, 104(51):20167-72, dec 2007.

[22] M. Lyon. A fast algorithm for Fourier continuation. SIAM J. Sci. Comput., 33(6):3241-3260, 2011.

[23] M. Lyon and O. P. Bruno. High-order unconditionally stable FC-AD solvers for general smooth domains II. Elliptic, parabolic and hyperbolic PDEs; theoretical considerations. Journal of Computational Physics, 229(9):3358-3381, may 2010.

[24] R. Matthysen and D. Huybrechs. Fast Algorithms for the computation of Fourier Extensions of arbitrary length. SIAM J. Sci. Comput., 36(2):828-845, 2015.

[25] A. Osipov, V. Rokhlin, and H. Xiao. Prolate Spheroidal Wave Functions of Order Zero, volume 187 of Applied Mathematical Sciences. Springer US, Boston, MA, 2013.

[26] R. B. Platte and A. Gelb. A Hybrid FourierChebyshev Method for Partial Differential Equations. Journal of Scientific Computing, 39(2):244-264, may 2009.

[27] H. Pollack and D. Slepian. Prolate spheroidal wave functions, Fourier analysis and uncertainty -III. Bell System Tech. J, 1961.

[28] H. Pollack and D. Slepian. Prolate spheroidal wave functions, Fourier analysis and uncertainty -IV. Bell System Tech. J, 1961.

[29] F. J. Simons and F. a. Dahlen. Spherical slepian functions and the polar gap in geodesy. Geophysical Journal International, 166(3):1039-1061, 2006.

[30] F. J. Simons and D. V. Wang. Spatiospectral concentration in the Cartesian plane. GEM International Journal on Geomathematics, 2(1):1-36, 2011.

[31] D. Slepian. Prolate spheroidal wave functions, Fourier analysis, and uncertainty -V: The Discrete Case. Bell Syst. Tech. J, 1978. 
[32] D. Slepian and H. Pollak. Prolate spheroidal wave functions, Fourier analysis and uncertaintyI. Bell System Technical Journal, 1961.

[33] A. V. Sobolev. Quasi-classical asymptotics for pseudodifferential operators with discontinuous symbols: Widom's conjecture. Functional Analysis and its Applications, 44(4):313-317, 2010.

[34] A. V. Sobolev. WienerHopf Operators in Higher Dimensions: The Widom Conjecture for PieceWise Smooth Domains. Integral Equations and Operator Theory, 81(3):435-449, 2015.

[35] H. Widom. On a Class of Integral Operators with Discontinuous Symbol, pages 477-500. Birkh\{ä\}user Basel, Basel, 1982.

[36] R. Wilson. Finite Prolate Spheroidal Sequences and their Applications 1: Generation and Properties. Pattern Analysis and Machine Intelligence, IEEE ..., (6):787-795, 1987.

[37] W. Xu and C. Chamzas. On the periodic discrete prolate spheroidal sequences. SIAM Journal on Applied Mathematics, 44(6):1210-1217, 1984. 